\title{
Self-Consistent Description of Multipole Strength in Exotic Nuclei I: Method
}

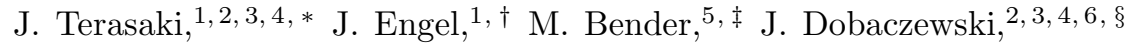 \\ W. Nazarewicz, ${ }^{2,3,6, \boldsymbol{T} \text { and M. Stoitsov }}{ }^{2,3,4,7, * *}$ \\ ${ }^{1}$ Department of Physics and Astronomy, CB3255, \\ University of North Carolina, Chapel Hill, NC 27599-3255 \\ ${ }^{2}$ Department of Physics and Astronomy, University of Tennessee, Knoxville, TN 37996 \\ ${ }^{3}$ Physics Division, Oak Ridge National Laboratory, P.O. Box 2008, Oak Ridge, TN 37831 \\ ${ }_{4}^{4}$ Joint Institute for Heavy-Ion Research, Oak Ridge, TN 37831 \\ ${ }^{5}$ Physics Division, Argonne National Laboratory, Argonne, IL 60439 \\ ${ }^{6}$ Institute of Theoretical Physics, Warsaw University, ul. Hoża 69, 00-681 Warsaw, Poland \\ ${ }^{7}$ Institute of Nuclear Research and Nuclear Energy, \\ Bulgarian Academy of Science, Sofia 1784, Bulgaria
}

(Dated: July 12, 2018)

\begin{abstract}
We use the canonical Hartree-Fock-Bogoliubov basis to implement a completely self-consistent quasiparticle-random-phase approximation with arbitrary Skyrme energy density functionals and density-dependent pairing functionals. The point of the approach is to accurately describe multipole strength functions in spherical even-even nuclei, including weakly-bound drip-line systems. We describe the method and carefully test its accuracy, particularly in handling spurious modes. To illustrate our approach, we calculate isoscalar and isovector monopole, dipole, and quadrupole strength functions in several $\mathrm{Sn}$ isotopes, both in the stable region and at the drip lines.
\end{abstract}

\section{INTRODUCTION}

The study of nuclei far from stability is an increasingly important part of nuclear physics [1, 2]. As radioactive beams allow more experiments on these nuclei, theoretical modeling is changing in significant ways. New ideas and progress in computer technology have allowed nuclear theorists to understand bits and pieces of nuclear structure quantitatively [3]. Short-lived exotic nuclei offer unique tests of those aspects of our developing many-body theories that depend on neutron excess [4]. The major challenge is to predict or describe in detail exotic new properties of nuclei far from the stability valley, and to understand the origins of these properties.

For medium-mass and heavy nuclei, an important goal is obtaining a universal energy-density functional, which will be able to describe static and dynamic properties of finite nuclei and extended nucleonic matter with arbitrary neutron-to-proton ratio. Self-consistent methods based on density-functional theory are already sophisticated enough to allow precise analysis of ground-state properties (e.g. binding energies) in heavy nuclei [5, 6, 7]. They can also help describe nuclear decays and excited states. Their predictions for collective excitations as we approach the neutron drip line are especially interesting. What happens to low- and high-frequency multipole modes when the neutron excess is unusually large?

To address these questions we use the quasiparticle random-phase approximation (QRPA), a powerful tool for understanding both low-lying vibrational states and giant resonances [8]. The QRPA is a microscopic approach that is nevertheless simple enough to allow "no-core" calculations. The approximation, which should be good for collective vibrations as long as their amplitudes are small, is especially effective in conjunction with Skyrme energy functionals. Our work is a part of a broad program to test and improve these functionals, which thus far have been fitted mainly to ground-state observables, by applying them to collective excitations, particularly near the drip line. This paper lays out our approach and evaluates its accuracy. For these purposes we restrict ourselves to a single Skyrme functional, $\mathrm{SkM}^{*}$. A forthcoming study will examine the performance of Skyrme functionals more generally.

The QRPA is a standard method for describing collective excitations in open-shell superconducting nuclei with stable mean-field solutions, either spherical or deformed. What is not standard, and at the same time is extremely important for weakly bound nuclei, is the treatment of the particle continuum. Continuum extensions of the random

\footnotetext{
*Electronic address: jterasak@physics.unc.edu

†Electronic address: engelj@physics.unc.edu

‡Electronic address: mbender@phy.anl.gov

$\S$ Electronic address: dobaczew@fuw.edu.pl

I Electronic address: witek@utk.edu

**Electronic address: stoitsovmv@ornl.gov
} 
phase approximation (RPA) or QRPA are usually carried out in coordinate space, facilitating treatment of decay channels and guaranteeing correct asymptotics. Surprisingly, as we discuss below, the rich literature on the RPA and QRPA, which includes many coordinate-space calculations, contains few treatments of the continuum that exploit the entire Skyrme functional in a fully self-consistent way.

To avoid confusion, we state what we mean by a fully self-consistent RPA or QRPA calculation. First, the underlying mean-field calculation must be self-consistent in the usual sense. Next, the residual interaction used in the RPA or QRPA must be derived from the same force or energy functional that determines the mean field. An important consequence of this condition, and of other more detailed technical conditions discussed below, is that spurious excitations arising from symmetry breaking by the mean field have zero or nearly zero energy, leaving the physical intrinsic excitations completely uncontaminated by spurious motion. Finally, energy-weighted sum rules must be satisfied to high accuracy. We elaborate on these requirements below; Refs. [9, 10, 11] discuss ways in which RPA calculations commonly violate them.

The literature applying RPA or QRPA to nuclear structure is huge, and a complete review is beyond the scope of our paper. We do, however, present an overview of the studies that are related in one way or another to nuclear density functionals, self consistency, pairing, and the key issue of the particle continuum.

The standard version of QRPA, the so-called matrix formulation, is carried out in the configuration space 12, 13. of single-quasiparticle states. A number of papers treat collective states in spherical nuclei in the Skyrme-RPA and QRPA matrix formulation (see Refs. 13, 14] and references cited therein), in which the positive-energy continuum is discretized, e.g. by solving the Hartree-Fock-Bogoliubov (HFB) and QRPA equations in a harmonic-oscillator singleparticle basis. Within this group, the first fully self-consistent calculations that properly account for continuum effects are those of Refs. 15, 16], in which the localized canonical basis of coordinate-space HFB is used to calculate betadecay rates of neutron-rich r-process nuclei and Gamow-Teller strength distributions. Recently, fully self-consistent HFB+QRPA calculations have also been carried out with the finite-range Gogny force [17. Unlike many previous Gogny+HFB studies that employed a harmonic oscillator basis, Ref. 17] solves the HFB equations in the eigenbasis of a Woods-Saxon potential, the particle continuum of which is discretized by enclosing the system in a box.

Coordinate-space Green's functions as a method of implementing the RPA through linear response were first used in Ref. 18 and subsequently applied to the description of low- and high-energy nuclear modes (see, e.g., Refs. 9, 19, 20, 21, 22, 23, 24, 25, 26, 27, 28]). Many of those calculations are not realistic enough, however, because they ignore the spin-orbit and Coulomb residual interactions in the RPA 10, 11]. Coordinate-space Green's-function QRPA was studied in Ref. [29], in the BCS approximation, with a phenomenological Woods-Saxon average potential. Coordinate-space HFB+QRPA for spherical nuclei was formulated in Refs. 30, 31, 32, 33] and applied to excitations of neutron-rich nuclei. As in [29], the Hartree-Fock (HF) field in Refs. 30, 31] was approximated by a Woods-Saxon potential. While the calculations of Refs. 32, 33 are based on Skyrme-HFB fields, they violate full self consistency by replacing the residual velocity-dependent terms of the Skyrme force by the Landau-Migdal force in the QRPA, and neglecting spin-spin, spin-orbit, and Coulomb residual interactions entirely. Within this approach, extensive Skyrme-HF+BCS QRPA calculations of E1-strength in neutron-rich nuclei were carried out in Refs. [34, 35].

An alternative coordinate-representation approach, also based on Green's functions, was formulated in Refs. 36, 37] within Migdal's finite-Fermi-systems theory. Most of practical applications of this method, however, involve approximations that break self consistency in one way or another, including the use of highly truncated pairing spaces, different interactions in HFB and QRPA, and the so-called diagonal pairing approximation 36, 38, 39, 40, 41, 42, 43, 44, 45. Properties of excited states and strength functions have also been investigated within the relativistic RPA [46, 47, 48, 49, 50, 51, 52] or QRPA [53, 54]. The QRPA work employs the matrix formulation and is fully self-consistent, since it uses the same Lagrangian in the relativistic Hartree-Bogoliubov calculation of the ground state and in the QRPA matrix equations, which are solved in the canonical basis.

At the present, no fully self-consistent continuum HFB+QRPA calculations exist in deformed nuclei. Refs. 55, 56. studied giant resonances in deformed nuclei within time-dependent HF theory, formulated in coordinate space with a complex absorbing boundary condition imposed. Symmetry-unrestricted RPA calculations, with no pairing, were carried out in Ref. [57] in a "mixed representation" [58] on a Cartesian mesh in a box, while Ref. [59] contains examples of BCS+QRPA calculations in the single-particle basis of a deformed Woods-Saxon potential.

The work described in this paper is fully self-consistent: among other things we use precisely the same interaction in the HFB and QRPA calculations so as to preserve the small-amplitude limit of time-dependent HFB. We formulate the QRPA in the canonical eigenbasis of the one-body particle-density matrix [60] which is calculated in the coordinate representation in a large spherical box. As mentioned above, the canonical basis has been used previously to study $\beta$ decay and Gamow-Teller strength [15, 16]; its use in charge-conserving modes near the drip line is more challenging, 
however, because of the existence of spurious states in the monopole and dipole channels. ${ }^{1}$ These zero-energy modes can mix with physical states unless the QRPA equations are solved with high accuracy. A less precise implementation of our approach was used to calculate neutrino-nucleus cross sections in ${ }^{208} \mathrm{~Pb}$ in Ref. 62].

This paper is organized as follows. Section पbelow presents our approach. In Sec. III we check the QRPA solutions carefully, focusing on spurious modes. Section [V contains the main conclusions of our work. Mathematical details are in two appendices, the first of which is on the QRPA equations and the second on calculating the derivatives of the Skyrme functionals that enter the formalism.

\section{METHOD}

Our first step in the self-consistent treatment of excitations is to solve the spherical HFB equations in coordinate space (without mixing neutron and proton quasiparticle wave functions [63]), with the method developed in Ref. [64] (see also Refs. 60, 65, 66]). We can use arbitrary Skyrme functionals in the particle-hole and pairing (particle-particle) channels.

We modify the code used in Refs. 60, 64, 65] so that it solves the HFB equations with higher accuracy, which we need because the QRPA uses all the single-quasiparticle states produced by the HFB equations, even those that are essentially unoccupied. Our modifications are: (i) the use of quadruple precision (though in solving the QRPA equations we use double precision); (ii) a smaller discretization length $(0.05 \mathrm{fm})$; and (iii) a high quasiparticle-energy cutoff $(200 \mathrm{MeV})$ and a maximum angular momentum $j_{\max }=15 / 2(N \leq 82)$ or $21 / 2(N>82)$. In a $20 \mathrm{fm}$ box, this cutoff corresponds to 200-300 quasiparticle states for each kind of nucleon. We include all these quasiparticle states in the HFB calculation because a very large energy cutoff is essential for the accuracy of self-consistent QRPA calculations [10]. Hence, the effective pairing window in our HFB calculations is also very large, with the pairing functional fitted to experimental pairing gaps extracted as in Ref. 67] from the measured odd-even mass differences in several $\mathrm{Sn}, \mathrm{Ni}$, and $\mathrm{Ca}$ isotopes.

Next, we construct the canonical basis, the eigenstates of single-particle density matrix $\rho$. To avoid poor accuracy (see Ref. [60]) in the wave functions of the nearly empty canonical particle states, we do not diagonalize $\rho$ directly in coordinate space. Instead we construct an intermediate basis by orthonormalizing a set of functions $\left\{\varphi_{1}^{\mu}(\boldsymbol{r})+\varphi_{2}^{\mu}(\boldsymbol{r})\right\}$, where $\varphi_{1}^{\mu}(\boldsymbol{r})$ and $\varphi_{2}^{\mu}(\boldsymbol{r})$ are the upper and lower components of the quasiparticle wave function with energy $E_{\mu}$ [64]. We use the density matrix in coordinate space to calculate the matrix in this basis, which we then diagonalize to obtain the canonical states. The reason for using the sum of $\varphi_{1}^{\mu}(\boldsymbol{r})$ and $\varphi_{2}^{\mu}(\boldsymbol{r})$ is that solutions of the HFB equations expressed in the canonical basis (Eqs. (4.14) of Ref. [60]) are, in the new basis, guaranteed to be numerically consistent with those of the original HFB problem. This is because the configuration space is the same in both cases, independent of the pairing cutoff (see Ref. [68] for a discussion relevant to this point). Without pairing, when either $\varphi_{1}^{\mu}(\boldsymbol{r})$ or $\varphi_{2}^{\mu}(\boldsymbol{r})$ is equal to zero, our method is equivalent to taking a certain number of HF states, including many unoccupied states.

In the canonical basis, the HFB+QRPA equations have a form almost identical to that of the BCS+QRPA approximation, the only difference being the presence of off-diagonal terms in the single-quasiparticle energies. The QRPA+HFB formalism employs more pairing matrix elements than the QRPA+BCS, however.

As noted already, full self consistency requires the use of the same interaction in the QRPA as in the HFB approximation. More specifically, this means that the matrix elements that enter the QRPA equation are related to second derivatives of a mean-field energy functional. We describe the densities and the form of the functional carefully in the appendices. But we must meet other conditions as well for QRPA calculation to be self-consistent. Essentially all the single-particle or quasiparticle states produced by the HFB calculation must be used in the space of two-quasiparticle QRPA excitations. This requirement is rather stringent, so we truncate the two-quasiparticle space at several levels and check for convergence of the QRPA solution. First we omit canonical-basis wave functions that have occupation probabilities $v_{i}^{2}$ less than some small $v_{\text {crit }}^{2}$, (or HF energies greater than some $\varepsilon_{\text {crit }}$ if there is no pairing). Then we exclude from the QRPA pairs of canonical states for which the occupation probabilities are both larger than $1-v_{\text {crit }}^{2}$. This second cut is based on the assumption that two-particle transfer modes are not strongly coupled to particle-hole excitations. In addition, if the factors containing $u_{i}$ and $v_{i}$ in the QRPA equation - see Eqs. (A12) and (A13) - are very small, in practice smaller than 0.01 , then we set the corresponding matrix elements equal to zero. This does not affect the size of the QRPA space, but significantly speeds up the calculations. For good performance we diagonalize QRPA-Hamiltonian matrices of order 20,000 × 20,000 in neutron-rich Sn isotopes.

\footnotetext{
${ }^{1}$ As far as we know, the only application of the canonical basis to charge-conserving modes near the drip line is in the relativistic QRPA, see e.g. [53, 61].
} 
Having solved the QRPA equations, we can then calculate the strength function

$$
S_{J}(E)=\frac{1}{\pi} \sum_{k} \sum_{M=-J}^{J} \frac{\gamma\left(E_{k}\right)\left|\left\langle k\left|\hat{F}_{J M}\right| 0\right\rangle\right|^{2}}{\left(E_{k}-E\right)^{2}+\gamma^{2}\left(E_{k}\right)},
$$

for the multipole operator $\hat{F}_{J M}$. The smoothing width $\gamma$ is supposed to be large enough to remove spurious oscillations in $S_{J}(E)$ associated with a finite box radius $R_{\text {box }}[55,69]$. A reasonable form, based on a single-particle estimate, for the smoothing width (App. B of Ref. [69]), is

$$
\gamma(E)=\left\{\begin{array}{ll}
\frac{\pi}{R_{\text {box }}} \sqrt{\frac{\hbar^{2}\left(E+\lambda_{\mathrm{n}}\right)}{2 m},}, & E \geq-\lambda_{\mathrm{n}} \\
0.1 \mathrm{MeV}, & E<-\lambda_{\mathrm{n}}
\end{array},\right.
$$

where $\lambda_{\mathrm{n}}$ is the neutron Fermi level and $m$ is the nucleon mass. In deriving Eq. (2) we assumed that the single-proton continuum is effectively shifted up several $\mathrm{MeV}$ by the Coulomb barrier. In other words, we associate the threshold energy with the neutron Fermi level.

In all the tests below, we use the Skyrme functional $\mathrm{SkM}^{*}[70$ and a volume pairing functional 71$]\left(C^{\tilde{\rho}}\left(\rho_{00}\right)\right.$ a constant in Eq. (B99)). The pairing parameter in Eq. (B19) is $V_{0}=-77.5 \mathrm{MeV} \mathrm{fm}{ }^{3}$. Usually we work in a box of radius $20 \mathrm{fm}$, though we vary this radius below to see its effects. In several tests we examine the weakly bound nucleus ${ }^{174} \mathrm{Sn}$, which is very close to the two-neutron drip line. In this system, the protons are unpaired and the neutrons paired (with $\Delta_{\mathrm{n}}=1.016 \mathrm{MeV}$ ) in the HFB ground state.

\section{ACCURACY OF SOLUTIONS}

Benchmark tests of the HFB part of our calculations are reported in Ref. 72]. Since the accuracy of the canonical wave functions, in which the QRPA calculations are carried out, strongly affects the quality of results (in particular QRPA self consistency), we take special care to compute them precisely. As discussed in Sec. III we obtain canonical states by diagonalizing the single-particle density matrix $\rho$ represented in the orthonormalized set of functions $\left\{\varphi_{1}^{\mu}(\boldsymbol{r})+\right.$ $\left.\varphi_{2}^{\mu}(\boldsymbol{r})\right\}$. The accuracy of this method is illustrated in Fig. 1 which plots the quasiparticle energies $E_{\mu}^{\text {check }}$, obtained by diagonalizing the HFB Hamiltonian in the canonical basis (Eq. (4.20) of Ref. 60]), versus the quasiparticle energies $E_{\mu}$ obtained by solving the HFB differential equations directly in coordinate space (Eq. (4.10) of Ref. [60]). Two sets of canonical states are used: (i) those obtained through the procedure outlined above (dotted line) and (ii) those obtained in the standard way by diagonalizing the density matrix $\rho\left(\boldsymbol{r}, \boldsymbol{r}^{\prime}\right)$ in discretized coordinate space (Eq. (3.24a) of Ref. [60]; solid line). If the canonical basis is precisely determined, $E_{\mu}^{\text {check }}=E_{\mu}$ and the two sets of $E_{\mu}^{\text {check }}$ coincide. Within the standard approach, however, the high canonical energies deviate visibly from their HFB counterparts, i.e., the accuracy of the underlying canonical wave functions is poor. On the other hand, the quasiparticle energies and canonical wave functions calculated within the modified approach introduced above are as accurate as the original solutions to the HFB equations, even for high-lying nearly-empty states. (See also Sec. VI.D of Ref. 73] for a discussion relevant to this point.)

Having examined the canonical basis, we turn to the accuracy of the QRPA part of the calculation. To test it, we first consider solutions related to symmetries. If a Hamiltonian is invariant under a symmetry operator $\hat{P}$ and the HFB state $|\Psi\rangle$ spontaneously breaks the symmetry, then $e^{i \alpha \hat{P}}|\Psi\rangle$, with $\alpha$ an arbitrary c-number, is degenerate with the state $|\Psi\rangle$. The QRPA equations have a spurious solution at zero energy associated with the symmetry breaking [8, 74], while all other solutions are free of the spurious motion. This property is important for strength functions, and gives us a way of testing the calculations. Since our QRPA equations, which assume spherical symmetry, are based on mean fields that include pairing and are localized in space, there appear spurious states associated with particle-number nonconservation (proton and/or neutron; $0^{+}$channel) and center-of-mass motion ( $1^{-}$channel). These two cases are discussed below in Sec. IIA and Sec. IIB

\section{A. The $0^{+}$isoscalar mode}

In addition to the spurious state associated with nonconservation of particle-number by the $\mathrm{HFB}$, the $0^{+}$channel contains the important "breathing mode". In Table \ we display results from a run with $v_{\text {crit }}^{2}=10^{-12}$ for neutrons and $\varepsilon_{\text {crit }}=150 \mathrm{MeV}$ for protons, resulting in the inclusion of 310 proton quasiparticle states and the same number of neutron states, with angular momentum up to $j=21 / 2$. The Table shows the QRPA energies and transition matrix elements of the particle-number operator. The spurious state is below $200 \mathrm{keV}$, well separated from the other 


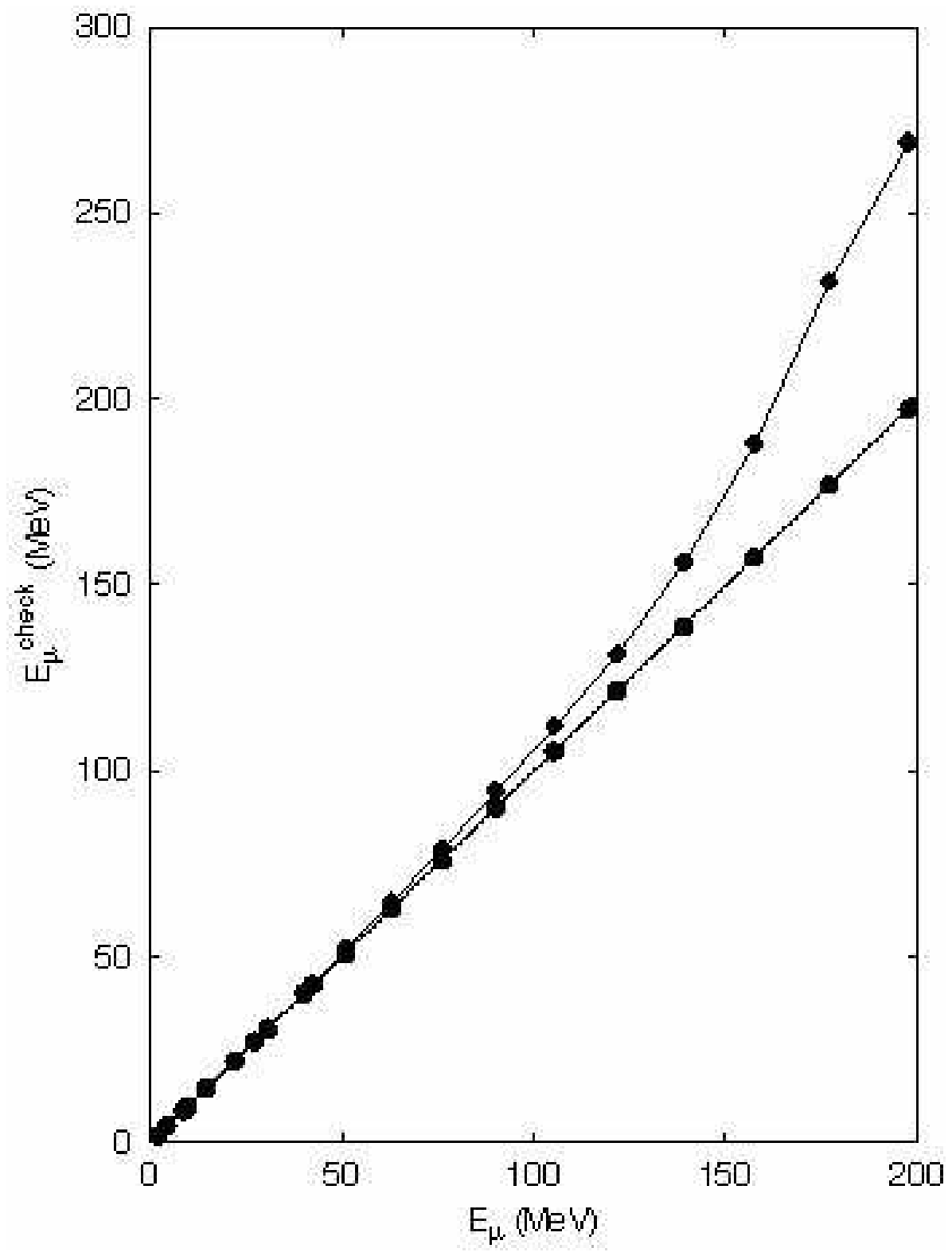

FIG. 1: Neutron quasiparticle energies $E_{\mu}^{\text {check }}$ for $s_{1 / 2}$ states in ${ }^{174} \mathrm{Sn}$, calculated by diagonalizing the HFB Hamiltonian in the canonical basis, versus the quasiparticle energies $E_{\mu}$ obtained by directly solving the HFB equations in coordinate space. Standard (solid line, diamonds) and improved (dotted line, dots) methods are used to obtain the canonical states. See text for details.

states, all of which have negligible "number-strength". The nonzero number strength in the spurious state, like the nonzero energy of that state, is a measure of numerical error. If the space of two-quasiparticle states is smaller, with

TABLE I: The lowest-energy excited $0^{+}$states in ${ }^{174} \mathrm{Sn}$. The second column shows the excitation energies and the third column the squared matrix elements of the particle-number operator between the $k$ th excited state and the ground state $(k=0)$.

\begin{tabular}{lll}
\hline \hline$k$ & $E_{k}(\mathrm{MeV})$ & $|\langle k|\hat{N}| 0\rangle|^{2}$ \\
\hline 1 & 0.171 & 0.120 \\
2 & 2.833 & $0.533 \times 10^{-5}$ \\
3 & 3.090 & $0.877 \times 10^{-7}$ \\
4 & 3.810 & $0.252 \times 10^{-5}$ \\
5 & 3.878 & $0.480 \times 10^{-5}$ \\
\hline \hline
\end{tabular}




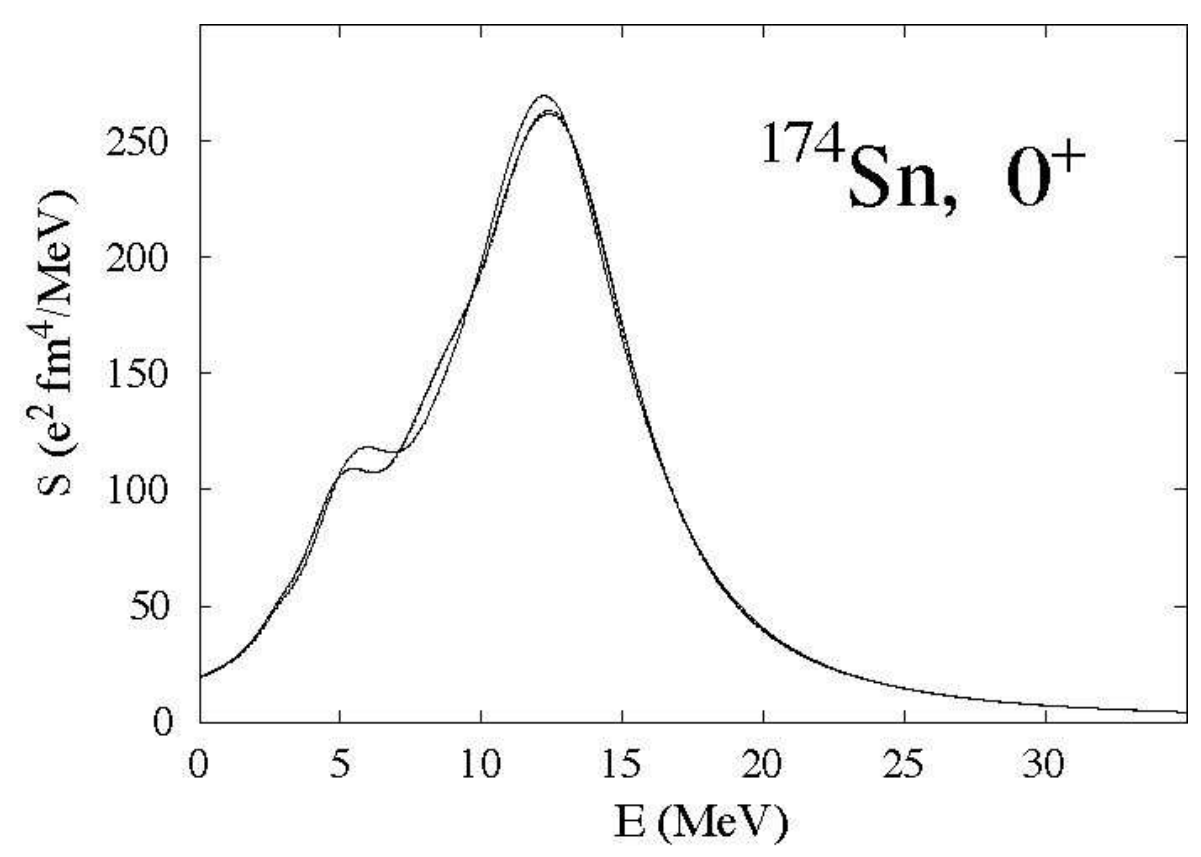

FIG. 2: Isoscalar $0^{+}$strength function in ${ }^{174} \mathrm{Sn}$ for (i) the single-proton energy cutoff $\varepsilon_{\text {crit }}=100 \mathrm{MeV}$ and the neutronquasiparticle occupation cutoff $v_{\text {crit }}^{2}=10^{-8}$ (thin solid line); (ii) $\varepsilon_{\text {crit }}=150 \mathrm{MeV}$ and $v_{\text {crit }}^{2}=10^{-12}$ (dotted line); and (iii) $\varepsilon_{\text {crit }}=200 \mathrm{MeV}$ and $v_{\text {crit }}^{2}=10^{-16}$ (thick solid line). Results corresponding to (ii) and (iii) practically coincide.

$\varepsilon_{\text {crit }}=100 \mathrm{MeV}$ and $v_{\text {crit }}^{2}=10^{-8}$, the energy of the spurious state and the number strength barely change.

Figure 2 shows the strength function $S_{J}(E)$ for the isoscalar $0^{+}$transition operator, cf. [75],

$$
\hat{F}_{00}=\frac{e Z}{A} \sum_{i=1}^{A} r_{i}^{2} .
$$

We have plotted three curves with successively more quasiparticle levels (from 246 proton levels and 203 neutron levels to 341 proton levels and 374 neutron levels), with cutoff parameters given in the figure caption. The major structures in the strength function are stable. The error remaining after to the gentlest truncation is extremely small.

The dependence of the strength function on the box size and quasiparticle cutoff is shown in Fig. 3 The upper part of the Figure (panels a-c) corresponds to a constant smoothing width of $\gamma=0.5 \mathrm{MeV}$. This relatively small value is not sufficient to eliminate the finite-box effects but it allows us to assess the stability of the QRPA solutions as a function of $R_{\text {box }}$. The large structure corresponding to the giant monopole resonance (GMR) is independent of box size no matter what the cutoff, but increasing the number of configurations magnifies the dependence on box size of local fluctuations in $S_{J}(E)$. The lower part of the Figure (panels d-f) are smoothed more realistically, as in Eq. (2). It is gratifying to see that the resulting strength functions are practically identical, i.e., the remaining dependence on $R_{\text {box }}$ and the cutoff is very weak.

The energy-weighted sum rule (EWSR) for the isoscalar $0^{+}$mode [75] is given by

$$
\sum_{k} E_{k}\left|\left\langle k\left|\hat{F}_{00}\right| 0\right\rangle\right|^{2}=2 \frac{e^{2} \hbar^{2}}{m} \frac{Z^{2}}{A}\left\langle r^{2}\right\rangle,
$$

where the expectation value is evaluated in the HFB ground state. This sum rule provides a stringent test of self consistency in the QRPA. In ${ }^{174} \mathrm{Sn}$, the right-hand side of Eq. (4) is $35215 e^{2} \mathrm{MeV} \mathrm{fm}{ }^{4}$ and the left-hand side $34985 \pm 15$ $e^{2} \mathrm{MeV} \mathrm{fm}^{4}$ for all of the calculations of Fig. 3 the QRPA strength essentially exhausts the sum rule. (The QRPA values of the EWSR in this paper are obtained by summing up to $E_{k}=50 \mathrm{MeV}$. )

\section{B. The isoscalar $1^{-}$mode}

The $1^{-}$channel, home of the giant dipole resonance, the isoscalar squeezing resonance, and as yet incompletely understood low-energy peaks in neutron-rich nuclei (sometimes associated with skin excitations), has a spurious 


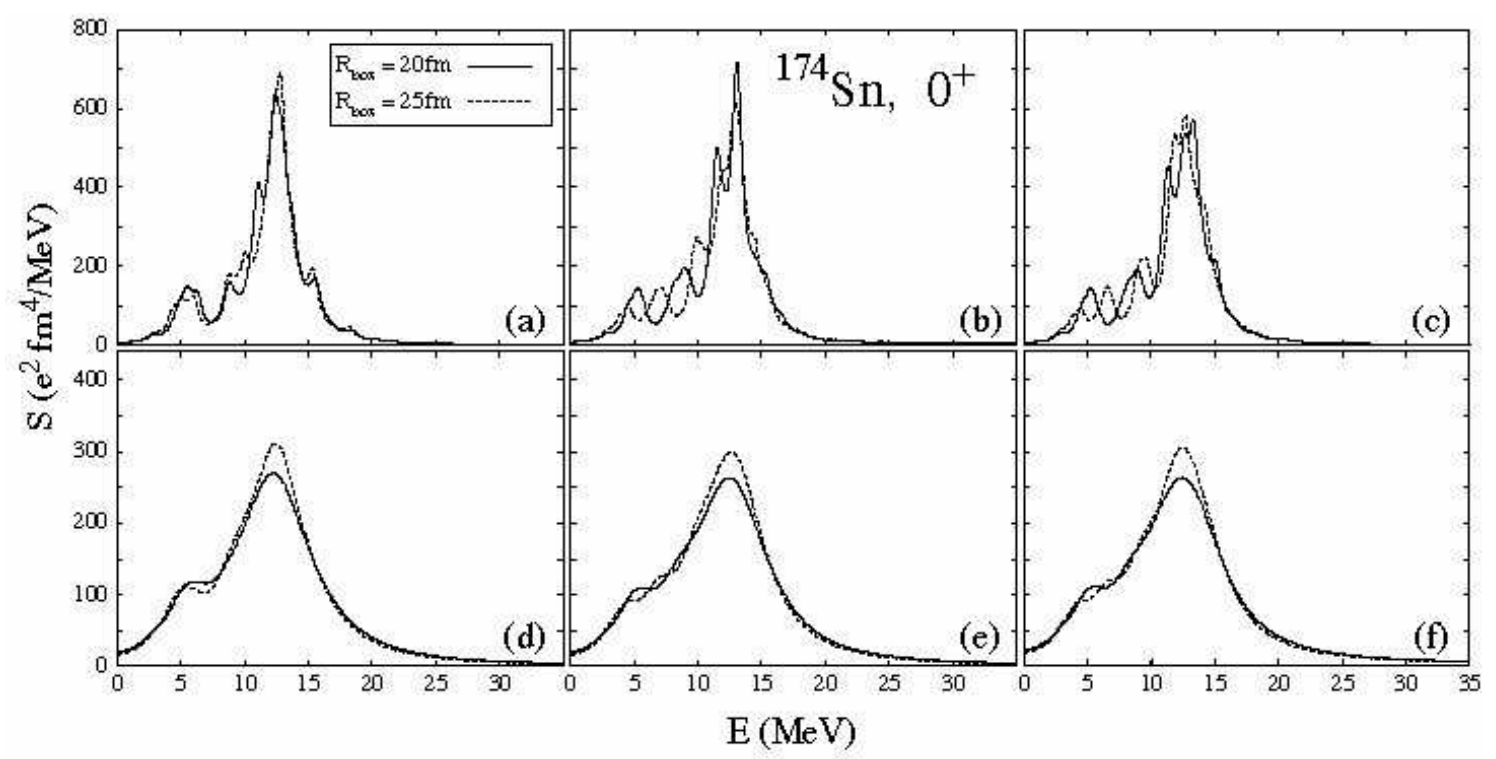

FIG. 3: Isoscalar $0^{+}$strength function in ${ }^{174} \mathrm{Sn}$ for the box radii: $R_{\mathrm{box}}=20 \mathrm{fm}$ (solid line) and $R_{\mathrm{box}}=25 \mathrm{fm}$ (dotted line). In (a), (b), and (c) the smoothing-width parameter $\gamma$ is 0.5 MeV for all energies, while in (d), (e), and (f) $\gamma(E)$ is given by Eq. (2). We use the same three sets of cutoff conditions as in Fig. 22 namely (i) in parts (a) and (d), (ii) in parts (b) and (e), and (iii) in parts (c) and (f).

isoscalar mode associated with center-of-mass motion that can seriously compromise the low-energy spectrum if not handled with extreme care. We test the ability of our QRPA to do so in ${ }^{100} \mathrm{Sn},{ }^{120} \mathrm{Sn},{ }^{174} \mathrm{Sn}$, and ${ }^{176} \mathrm{Sn}$. (The nuclei ${ }^{100} \mathrm{Sn}$ and ${ }^{176} \mathrm{Sn}$ are the two-proton and two-neutron drip-line systems predicted by the HFB calculation with SkM*.

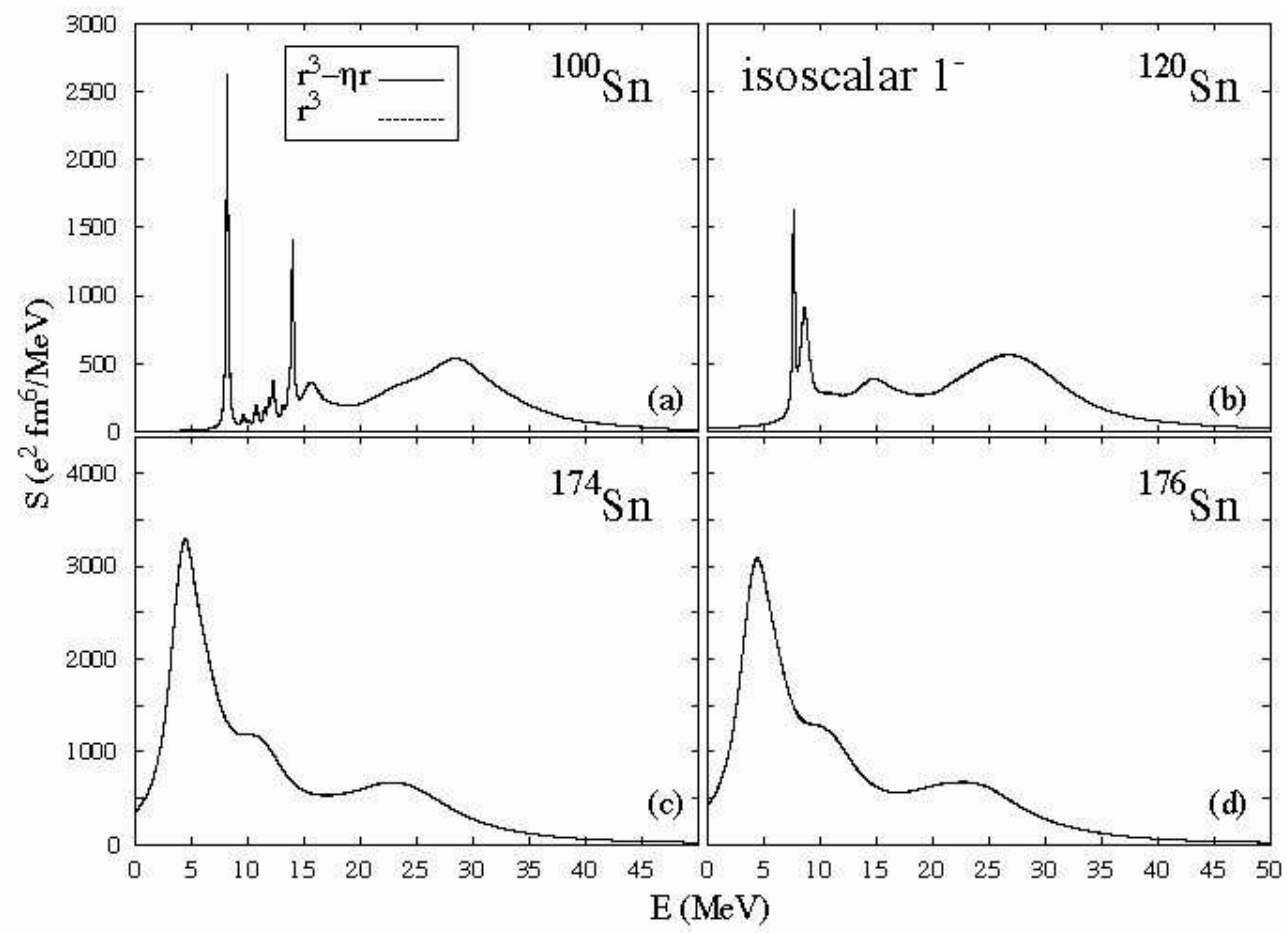

FIG. 4: Isoscalar $1^{-}$strength function in ${ }^{100,120,174,176} \mathrm{Sn}$ for the corrected dipole operator in Eq. (6) (solid line) and the uncorrected operator in Eq. (5) (dotted line). The cutoff $\varepsilon_{\text {crit }}$ is $140 \mathrm{MeV}$ and $v_{\text {crit }}^{2}$ is $3 \times 10^{-12}$. The self consistency of our calculations makes the solid and dotted curves coincide nearly exactly over the whole energy range. 
Neither nucleus has any static pairing, i.e., $\Delta_{n}=\Delta_{p}=0$.) In the following calculations, we take $\varepsilon_{\text {crit }}=140 \mathrm{MeV}$ for the protons and $v_{\text {crit }}^{2}=9 \times 10^{-12}$ for the neutrons. As discussed above, smoothed strength functions are practically independent of small changes in the cutoff. They are also independent of the cutoff in quasiparticle angular momentum provided we include all states with $j \leq 15 / 2$.

Figure 4 shows the predicted isoscalar dipole strength function for ${ }^{100,120,174,176} \mathrm{Sn}$. For the transition operator, we use

$$
\hat{F}_{1 M}=\frac{e Z}{A} \sum_{i=1}^{A} r_{i}^{3} Y_{1 M}\left(\Omega_{i}\right)
$$

and the corrected operator

$$
\hat{F}_{1 M}^{\mathrm{cor}}=\frac{e Z}{A} \sum_{i=1}^{A}\left(r_{i}^{3}-\eta r_{i}\right) Y_{1 M}\left(\Omega_{i}\right), \quad \eta=\frac{5}{3}\left\langle r^{2}\right\rangle,
$$

to remove as completely as possible residual pieces of the spurious state from the physical states [10]. The fact that the strength functions produced by these two operators - displayed in Fig. 4 - coincide so closely shows the extreme accuracy of our QRPA solutions; they are uncontaminated by spurious motion even without the operator correction. The spurious-state energies $E_{\text {spurious }}$ are $0.964 \mathrm{MeV}$ for ${ }^{100} \mathrm{Sn}$ and $0.713 \mathrm{MeV}$ for ${ }^{120} \mathrm{Sn}$, and the energies of the first physical excited states are $7.958 \mathrm{MeV}$ for ${ }^{100} \mathrm{Sn}$ and $7.729 \mathrm{MeV}$ for ${ }^{120} \mathrm{Sn}$. In ${ }^{174} \mathrm{Sn}\left({ }^{176} \mathrm{Sn}\right), E_{\text {spurious }}$ is $0.319 \mathrm{MeV}$ $(0.349 \mathrm{MeV})$ and the first physical state is at $3.485 \mathrm{MeV}(2.710 \mathrm{MeV})$, lower than in the more stable isotopes. Pairing correlations do not affect accuracy; the neutrons in ${ }^{120} \mathrm{Sn}$ and ${ }^{174} \mathrm{Sn}$ are paired, while those in ${ }^{100} \mathrm{Sn}$ and ${ }^{176} \mathrm{Sn}$ are not.

We display the fine structure of the isoscalar $1^{-}$strength functions in ${ }^{120} \mathrm{Sn}$ and ${ }^{174} \mathrm{Sn}$ in Fig. 5 which also illustrates the dependence of the results on $R_{\mathrm{box}}$. The dependence is consistent with that of Fig. 3 for the isoscalar $0^{+}$strength;

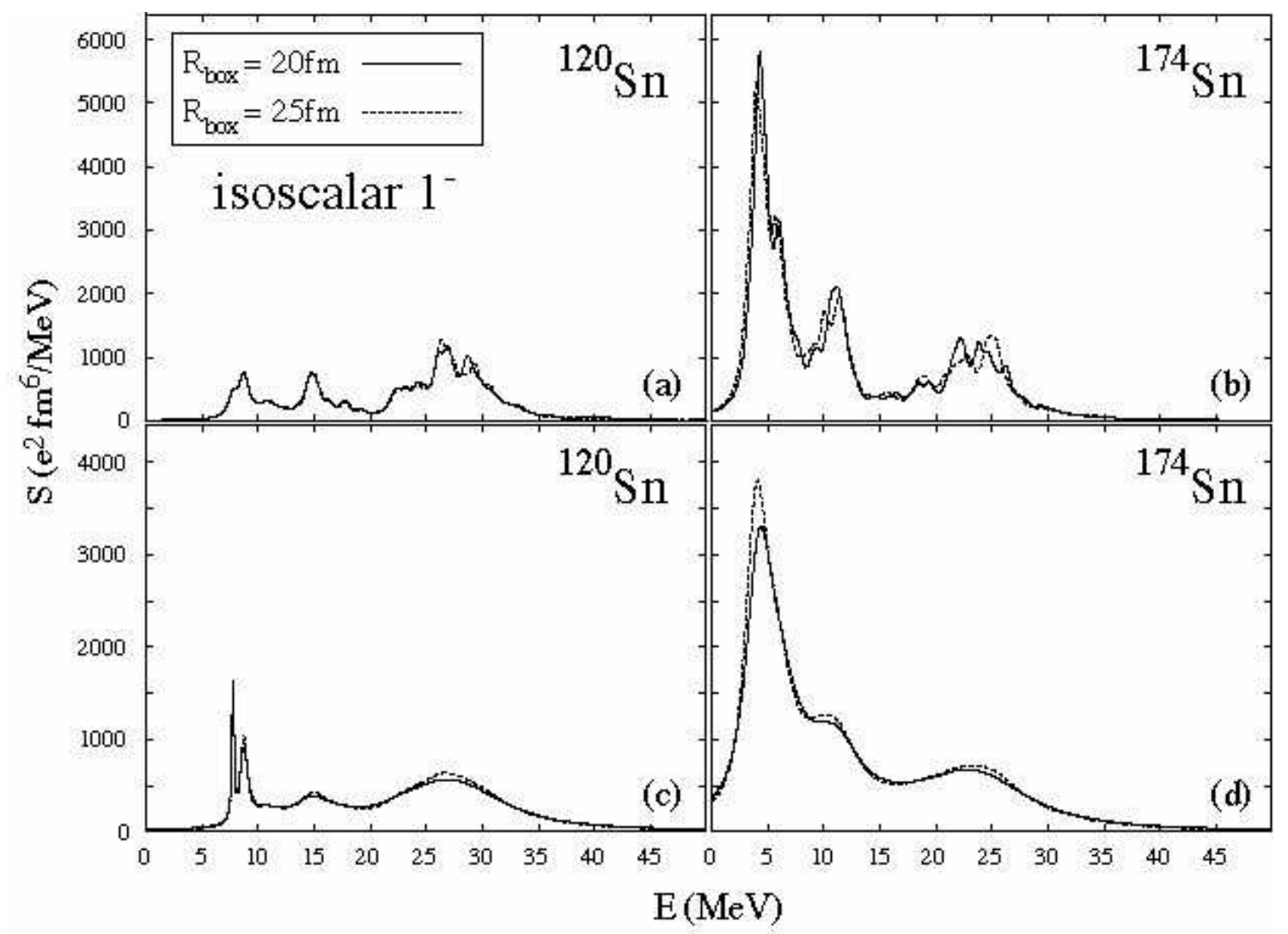

FIG. 5: $\quad$ Isoscalar $\quad 1^{-}$strength function in ${ }^{120} \mathrm{Sn} \quad$ (left) and ${ }^{174} \mathrm{Sn} \quad$ (right) for two box radii: $\quad R_{\text {box }}=20 \mathrm{fm}$ (solid line) and $R_{\text {box }}=25 \mathrm{fm} \quad$ (dotted line). In (a) and (b) the smoothingwidth parameter is constant $(\gamma=0.5 \mathrm{MeV})$, while in (c) and (d) $\gamma(E)$ is given by Eq. (2). 
the low-amplitude fluctuations in $S_{J}(E)$ that are unstable as a function of $R_{\text {box }}$ disappear, and the smoothed strength function depends only weakly on $R_{\mathrm{box}}$. In ${ }^{120} \mathrm{Sn}$, the two sharp peaks below $10 \mathrm{MeV}$ correspond to discrete states while the broad maxima centered around $15 \mathrm{MeV}$ and $27 \mathrm{MeV}$ are in the continuum, well above neutron-emission threshold. A similar three-peaked structure emerges in ${ }^{174} \mathrm{Sn}$, though most of the strength there is concentrated in the low-energy peak at $E \approx 4 \mathrm{MeV}$. Fig. [4 shows (as we will discuss in our forthcoming paper [76]) that the appearance of the low-energy isoscalar dipole strength is a real and dramatic feature of neutron-rich dripline nuclei [77, 78]. The EWSR for the isoscalar $1^{-}$mode [75] is

$$
\sum_{k} \sum_{M} E_{k}\left|\left\langle k\left|\hat{F}_{1 M}^{\mathrm{cor}}\right| 0\right\rangle\right|^{2}=\frac{3}{8 \pi} \frac{e^{2} \hbar^{2}}{m} \frac{Z^{2}}{A}\left(11\left\langle r^{4}\right\rangle-\frac{25}{3}\left\langle r^{2}\right\rangle^{2}\right)
$$

In ${ }^{174} \mathrm{Sn}$, the right-hand side is $403310 e^{2} \mathrm{MeV} \mathrm{fm}{ }^{6}$, while the left-hand side is $400200 e^{2} \mathrm{MeV} \mathrm{fm}{ }^{6}$. For ${ }^{176} \mathrm{Sn}$, the corresponding numbers are $406576 e^{2} \mathrm{MeV} \mathrm{fm}^{6}$ and $407100 e^{2} \mathrm{MeV} \mathrm{fm}^{6}$. This level of agreement is very good.

\section{The isovector $0^{+}$and isoscalar, isovector $2^{+}$modes}

Figure 6 displays strength functions for the $0^{+}$and $2^{+}$channels in ${ }^{120} \mathrm{Sn}$ and ${ }^{174} \mathrm{Sn}$. (We discussed the isoscalar $0^{+}$ mode above to illustrate the accuracy of our solutions, but include it here as well for completeness.) The calculations show the appearance of low-energy $0^{+}$strength - both isovector and isoscalar - and low-energy isovector $2^{+}$strength in ${ }^{174} \mathrm{Sn}$, though in none of these instances is the phenomenon quite as dramatic as in the isoscalar $1^{-}$channel.

The EWSR for the isoscalar $2^{+}$transition operator,

$$
\hat{F}_{2 M}=e \frac{Z}{A} \sum_{i=1}^{A} r_{i}^{2} Y_{2 M}\left(\Omega_{i}\right),
$$

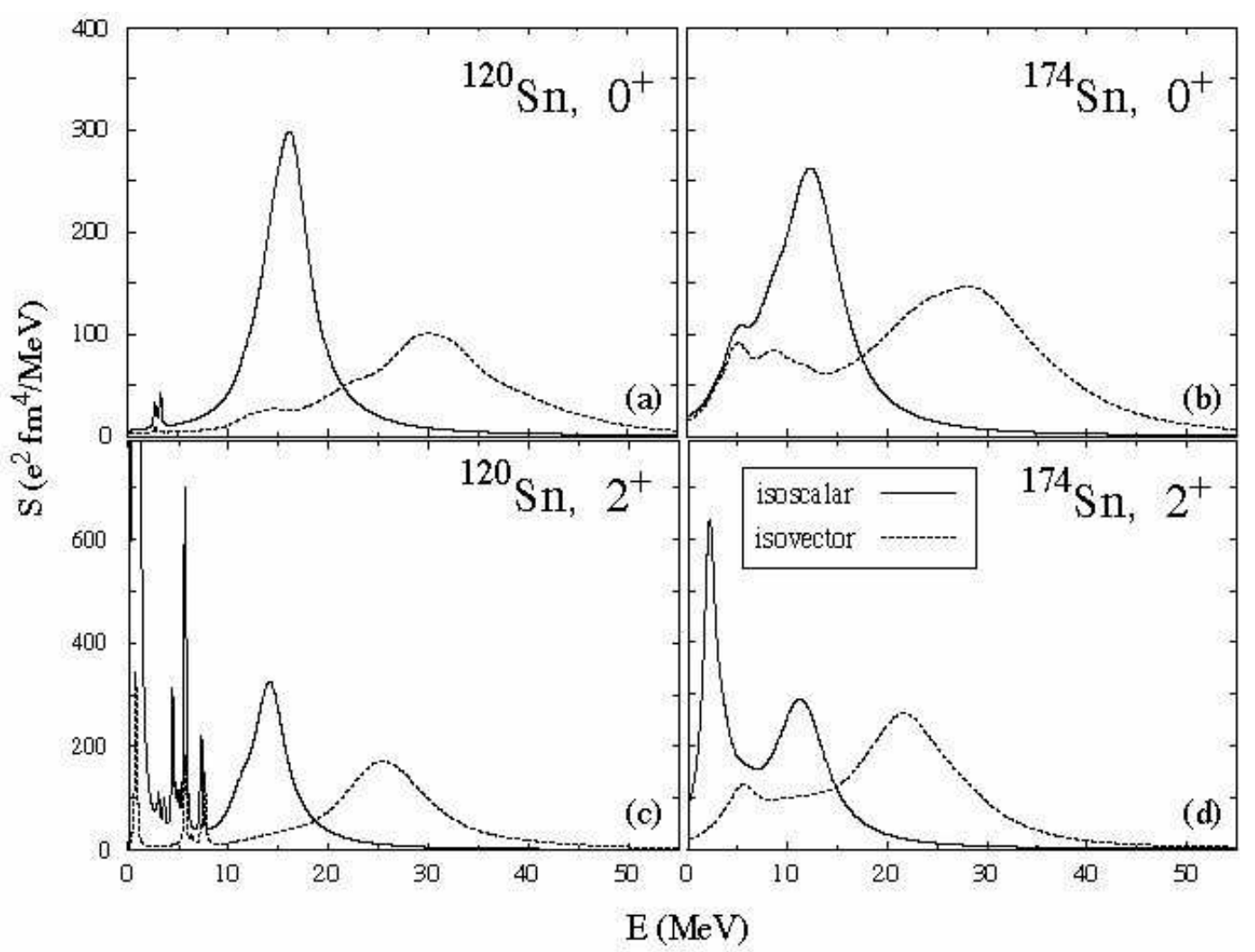

FIG. 6: Isoscalar and isovector strength functions for (a) the $0^{+}$channel of ${ }^{120} \mathrm{Sn}$, (b) the $0^{+}$channel of ${ }^{174} \mathrm{Sn}$, (c) the $2^{+}$channel of ${ }^{120} \mathrm{Sn}$, and (d) the $2^{+}$channel of ${ }^{174} \mathrm{Sn}$. The cutoff $\epsilon_{\text {crit }}$ is $150 \mathrm{MeV}$ and $v_{\text {crit }}^{2}$ is $10^{-12}$. 
can be written as 75

$$
\sum_{k} \sum_{M} E_{k}\left|\left\langle k\left|\hat{F}_{2 M}\right| 0\right\rangle\right|^{2}=\frac{25}{4 \pi} \frac{e^{2} \hbar^{2}}{m} \frac{Z^{2}}{A}\left\langle r^{2}\right\rangle .
$$

The sum rule is obeyed as well in the $2^{+}$isoscalar channel as in the $0^{+}$and $1^{-}$channels, the only difference being that one needs to include quasiparticle states with $j>15 / 2$ for ${ }^{174} \mathrm{Sn}$. For ${ }^{120} \mathrm{Sn}\left({ }^{174} \mathrm{Sn}\right)$ from Fig. 6 the EWSR is 37222 (34971) $e^{2} \mathrm{MeV} \mathrm{fm}^{4}$ while the QRPA value is 37030 (35010) $e^{2} \mathrm{MeV} \mathrm{fm}^{4}$.

While on the topic of the sum rule, we display in Table II the $j_{\text {max }}$-dependence of the EWSR for several channels in ${ }^{150} \mathrm{Sn}$, with $R_{\text {box }}=25 \mathrm{fm}$. By taking $j_{\max }=19 / 2$ we appear to obtain essentially the entire strength in all three cases.

TABLE II: The $j_{\max }$-dependence of isoscalar EWSR for ${ }^{150} \mathrm{Sn} . R_{\text {box }}$ is $25 \mathrm{fm}$.

\begin{tabular}{lcrr}
\hline \hline$T J^{\pi}$ & units & $j_{\max }=19 / 2$ & $j_{\max }=25 / 2$ \\
\hline IS $0^{+}$ & $\left(e^{2} \mathrm{MeV} \mathrm{fm}^{4}\right)$ & 35731 & 35633 \\
${\text { IS } 1^{-}}^{+}$ & $\left(e^{2} \mathrm{MeV} \mathrm{fm}^{6}\right)$ & 361686 & 353936 \\
IS $^{+}$ & $\left(e^{2} \mathrm{MeV} \mathrm{fm}^{4}\right)$ & 35542 & 35445 \\
\hline \hline
\end{tabular}

\section{CONCLUSION}

In this work we have reported on the development and detailed testing of a fully self-consistent Skyrme-QRPA framework that employs the canonical HFB basis. The method can be used to calculate strength distributions in any spin-isospin channel and in any spherical even-even nucleus. A good calculation requires a large single-quasiparticle space. Our results show that our space is large enough in nuclei as heavy as the Sn isotopes.

We are currently investigating the predictions of a range of Skyrme functionals across the Ca, Ni, and Sn isotope chains. The initial results presented here point to increases in low-lying strength at the neutron drip line, particularly in the isoscalar-dipole channel. In a forthcoming paper [76] we will report on the robustness of these effects, on the physics underlying them, on their variation with atomic mass and number, and on their implications for the future of Skyrme functionals.

\section{Acknowledgments}

We gratefully acknowledge useful discussions with Shalom Shlomo, Nils Paar, Dario Vretenar, and the Japanese members of the US-Japan cooperative project on "Mean-Field Approach to Collective Excitations in Unstable Medium-Mass and Heavy Nuclei". MB is grateful for the warm hospitality at the Physics Division at ORNL and the Theory Division at GSI Darmstadt, where most of his contribution to this work was made. This work was supported in part by the U.S. Department of Energy under Contracts Nos. DE-FG02-97ER41019 (University of North Carolina at Chapel Hill), DE-FG02-96ER40963 (University of Tennessee), DE-AC05-00OR22725 with UT-Battelle, LLC (Oak Ridge National Laboratory), DE-FG05-87ER40361 (Joint Institute for Heavy Ion Research), and W-31-109-ENG-38 (Argonne National Laboratory); by the National Science Foundation Contract No. 0124053 (U.S.-Japan Cooperative Science Award); by the Polish Committee for Scientific Research (KBN); and by the Foundation for Polish Science (FNP). We used the parallel computers of The Center for Computational Sciences at Oak Ridge National Laboratory and Information Technology Services at the University of North Carolina at Chapel Hill.

\section{APPENDIX A: QRPA EQUATION}

The QRPA equations are the small-oscillations limit of the time-dependent Hartree-Fock-Bogoliubov approximation, see, e.g., [8, 13]. In the canonical basis the most general equations take the form

$$
\sum_{L<L^{\prime}}\left(\begin{array}{cc}
A_{K K^{\prime}, L L^{\prime}} & B_{K K^{\prime}, L L^{\prime}} \\
-B_{K K^{\prime}, L L^{\prime}}^{*} & -A_{K K^{\prime}, L L^{\prime}}^{*}
\end{array}\right)\left(\begin{array}{c}
X_{L L^{\prime}}^{k} \\
Y_{L L^{\prime}}^{k}
\end{array}\right)=E_{k}\left(\begin{array}{c}
X_{K K^{\prime}}^{k} \\
Y_{K K^{\prime}}^{k}
\end{array}\right), \quad K<K^{\prime}
$$




$$
\begin{aligned}
& A_{K K^{\prime}, L L^{\prime}}=E_{K L} \delta_{K^{\prime} L^{\prime}}-E_{K^{\prime} L} \delta_{K L^{\prime}}-E_{K L^{\prime}} \delta_{K^{\prime} L}+E_{K^{\prime} L^{\prime}} \delta_{K L} \\
& -\bar{V}_{K \bar{L} \bar{K}^{\prime} L^{\prime}}^{\mathrm{ph}} u_{L^{\prime}} v_{L} u_{K} v_{K^{\prime}}+\bar{V}_{K^{\prime} \bar{L} \bar{K} L^{\prime}}^{\mathrm{ph}} u_{L^{\prime}} v_{L} u_{K^{\prime}} v_{K} \\
& +\bar{V}_{K \bar{L}^{\prime} \bar{K}^{\prime} L}^{\mathrm{ph}} u_{L} v_{L^{\prime}} u_{K} v_{K^{\prime}}-\bar{V}_{K^{\prime} \bar{L}^{\prime} \bar{K} L}^{\mathrm{ph}} u_{L} v_{L^{\prime}} u_{K^{\prime}} v_{K}
\end{aligned}
$$

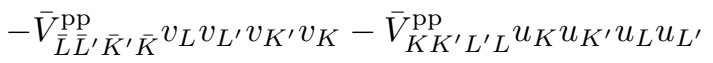

$$
\begin{aligned}
& -\bar{V}_{\bar{L}^{\prime} \bar{L}^{\prime} K \bar{K}^{\prime}}^{3 \mathrm{p} 1 \mathrm{~h}} v_{L} v_{L^{\prime}} u_{K} v_{K^{\prime}}+\bar{V}_{\bar{L}^{\prime} \bar{L}^{\prime} K^{\prime} \bar{K}^{3}}^{3 \mathrm{p} 1 \mathrm{~h}} v_{L} v_{L^{\prime}} u_{K^{\prime}} v_{K} \\
& -\bar{V}_{K K^{\prime} \bar{L} L^{\prime}}^{3 \mathrm{p} 1 \mathrm{~h}} u_{K} u_{K^{\prime}} u_{L^{\prime}} v_{L}+\bar{V}_{K K^{\prime} \bar{L}^{\prime} L}^{3 \mathrm{p} 1 \mathrm{~h}} u_{K} u_{K^{\prime}} u_{L} v_{L^{\prime}} \\
& -\bar{V}_{\bar{L} L^{\prime} \bar{K}^{\prime} \bar{K}^{1}}^{1 \mathrm{p} 3 \mathrm{~h}} u_{L^{\prime}} v_{L} v_{K^{\prime}} v_{K}+\bar{V}_{\bar{L}^{\prime} L \bar{K}^{\prime} \bar{K}}^{1 \mathrm{p} 3 \mathrm{~h}} u_{L} v_{L^{\prime}} v_{K^{\prime}} v_{K} \\
& -\bar{V}_{K \bar{K}^{\prime} L^{\prime} L}^{1 \mathrm{p} 3 \mathrm{~h}} u_{K} v_{K^{\prime}} u_{L} u_{L^{\prime}}+\bar{V}_{K^{\prime} \bar{K} L^{\prime} L}^{1 \mathrm{p} 3 \mathrm{~h}} u_{K^{\prime}} v_{K} u_{L} u_{L^{\prime}}, \\
& B_{K K^{\prime}, L L^{\prime}}=\bar{V}_{K^{\prime} L^{\prime} \bar{K} \bar{L}}^{\mathrm{ph}} u_{L^{\prime}} v_{L} u_{K^{\prime}} v_{K}-\bar{V}_{K L^{\prime} \bar{K}^{\prime} \bar{L}}^{\mathrm{ph}} u_{L^{\prime}} v_{L} u_{K} v_{K^{\prime}} \\
& -\bar{V}_{K^{\prime} L \bar{K} \bar{L}^{\prime}}^{\mathrm{ph}} u_{L} v_{L^{\prime}} u_{K^{\prime}} v_{K}+\bar{V}_{K L \bar{K}^{\prime} \bar{L}^{\prime}}^{\mathrm{ph}} u_{L} v_{L^{\prime}} u_{K} v_{K^{\prime}} \\
& +\bar{V}_{K^{\prime} K \bar{L} \bar{L}^{\prime}}^{\mathrm{pp}} v_{L} v_{L^{\prime}} u_{K} u_{K^{\prime}}+\bar{V}_{L^{\prime} L \bar{K} \bar{K}^{\prime}}^{\mathrm{pp}} v_{K} v_{K^{\prime}} u_{L} u_{L^{\prime}} \\
& +\bar{V}_{K^{\prime} K L^{\prime} \bar{L}}^{3 \mathrm{p} 1 \mathrm{~h}} u_{L^{\prime}} v_{L} u_{K} u_{K^{\prime}}-\bar{V}_{K^{\prime} K L \bar{L}^{\prime}}^{3 \mathrm{p} 1 \mathrm{~h}} u_{L} v_{L^{\prime}} u_{K} u_{K^{\prime}} \\
& +\bar{V}_{L^{\prime} L K^{\prime} \bar{K}}^{3 \mathrm{p} 1 \mathrm{~h}} u_{K^{\prime}} v_{K} u_{L} u_{L^{\prime}}-\bar{V}_{L^{\prime} L K \bar{K}^{\prime}}^{3 \mathrm{p} 1 \mathrm{~h}} u_{K^{\prime}} v_{K^{\prime}} u_{L} u_{L^{\prime}} \\
& +\bar{V}_{K^{\prime} \bar{K} \bar{L} \bar{L}^{\prime}}^{1 \mathrm{p} 3 \mathrm{~h}} v_{L} v_{L^{\prime}} u_{K^{\prime}} v_{K}-\bar{V}_{K \bar{K}^{\prime} \bar{L} \bar{L}^{\prime}}^{1 \mathrm{p} 3 \mathrm{~h}} v_{L} v_{L^{\prime}} u_{K} v_{K^{\prime}} \\
& +\bar{V}_{L^{\prime} \bar{L} \bar{K} \bar{K}^{\prime}}^{1 \mathrm{p} 3 \mathrm{~h}} v_{K} v_{K^{\prime}} u_{L^{\prime}} v_{L}-\bar{V}_{L \bar{L}^{\prime} \bar{K} \bar{K}^{\prime}}^{1 \mathrm{p} 3 \mathrm{~h}}, v_{K} v_{K^{\prime}} u_{L} v_{L^{\prime}}, \\
& \bar{V}_{K L K^{\prime} L^{\prime}}^{\mathrm{ph}}=\frac{\delta^{2} E\left[\rho, \kappa, \kappa^{*}\right]}{\delta \rho_{K^{\prime} K} \delta \rho_{L^{\prime} L}}, \\
& \bar{V}_{K^{\prime} K L^{\prime} L}^{\mathrm{pp}}=\frac{\delta^{2} E\left[\rho, \kappa, \kappa^{*}\right]}{\delta \kappa_{K^{\prime} K}^{*} \delta \kappa_{L^{\prime} L}}, \\
& \bar{V}_{K^{\prime} K L^{\prime} L}^{3 \mathrm{p} 1 \mathrm{~h}}=\frac{\delta^{2} E\left[\rho, \kappa, \kappa^{*}\right]}{\delta \kappa_{K^{\prime} K}^{*} \delta \rho_{L L^{\prime}}}=\bar{V}_{L L^{\prime} K^{\prime} K}^{1 \mathrm{p} 3 \mathrm{~h} *},
\end{aligned}
$$

where $K$ and $L$ are single-particle indices for the canonical basis, and the states are assumed to be ordered. The symbol $\bar{K}$ refers to the conjugate partner of $K, u_{K}$ and $v_{K}$ come from the BCS transformation associated with the canonical basis, and the $E_{K L}$ are the one-quasiparticle matrix elements of the HFB Hamiltonian (cf. Eq. (4.14b) of Ref. [60]). $X_{L L^{\prime}}^{k}$ and $Y_{L L^{\prime}}^{k}$ are the forward and backward amplitudes of the QRPA solution $k$, and $E_{k}$ is the corresponding excitation energy. $E\left[\rho, \kappa, \kappa^{*}\right]$ is the energy functional (see App. B for an explicit definition) and $\rho$ and $\kappa$ are the density matrix and pairing tensor. After taking the functional derivatives, we replace $\rho$ and $\kappa$ by their HFB solutions, in complete analogy with an ordinary Taylor-series expansion.

To write the equations in coupled form, we introduce the notation

$$
K \equiv\left(n_{\mu} l_{\mu} j_{\mu} m_{\mu}\right) \equiv\left(\mu m_{\mu}\right), \quad L \equiv\left(\nu m_{\nu}\right)
$$

where $(\mathrm{nljm})$ denote spherical quantum numbers. Using (i) rotational, time-reversal, and parity symmetries of the HFB state, (ii) the conjugate single-particle state ${ }^{2}$

$$
|\bar{K}\rangle=\left|\overline{\mu m_{\mu}}\right\rangle=(-)^{j_{\mu}-m_{\mu}}\left|\mu-m_{\mu}\right\rangle
$$

\footnotetext{
2 The conjugate state in our HFB code is slightly different: $|\overline{\mu m}\rangle=(-)^{j-m+l}|\mu-m\rangle$. This definition follows from Eq. (3.24b) of Ref. 60 and the single-particle wave function $\psi_{\mu m}(\boldsymbol{r})=R_{\mu}(r) \sum_{l_{z} \sigma} Y_{l l_{z}}(\Omega)\left\langle l l_{z} \frac{1}{2} \sigma \mid j m\right\rangle|\sigma\rangle$, where $R_{\mu}(r)$ and $|\sigma= \pm 1 / 2\rangle$ are real radial and spin wave functions. Thus, we multiply all HFB $v_{\mu}$ by $(-)^{l}$ in the QRPA calculations.
} 
and (iii) the relations

$$
\begin{aligned}
& X_{K K^{\prime}}^{k}=\left\langle j_{\mu} m_{\mu} j_{\mu^{\prime}} m_{\mu^{\prime}} \mid J_{k} M_{k}\right\rangle \bar{X}_{\left[\mu \mu^{\prime}\right] J_{k}}^{k} \times\left\{\begin{array}{cc}
\sqrt{2}, & \mu=\mu^{\prime}, \\
1, & \text { otherwise, }
\end{array}\right. \\
& Y_{K K^{\prime}}^{k}=(-)^{j_{\mu}-m_{\mu}}(-)^{j_{\mu^{\prime}}-m_{\mu^{\prime}}}\left\langle j_{\mu}-m_{\mu} j_{\mu^{\prime}}-m_{\mu^{\prime}} \mid J_{k} M_{k}\right\rangle \bar{Y}_{\left[\mu \mu^{\prime}\right] J_{k}}^{k} \times\left\{\begin{array}{cc}
\sqrt{2}, & \mu=\mu^{\prime}, \\
1, & \text { otherwise, }
\end{array}\right.
\end{aligned}
$$

with $J_{k}$ the angular momentum of the state $k$ and the factor $\sqrt{2}$ for convenience [12], one can rewrite the QRPA equation as

$$
\begin{aligned}
& \sum_{\nu \leq \nu^{\prime}}\left(\begin{array}{cc}
A_{\left[\mu \mu^{\prime}\right] J_{k},\left[\nu \nu^{\prime}\right] J_{k}} & B_{\left[\mu \mu^{\prime}\right] J_{k},\left[\bar{\nu} \bar{\nu}^{\prime}\right] J_{k}} \\
-B_{\left[\mu \mu^{\prime}\right] J_{k},\left[\bar{\nu} \bar{\nu}^{\prime}\right] J_{k}}^{*} & -A_{\left[\mu \mu^{\prime}\right] J_{k},\left[\nu \nu^{\prime}\right] J_{k}}^{*}
\end{array}\right)\left(\begin{array}{c}
\bar{X}_{\left[\nu \nu^{\prime}\right] J_{k}}^{k} \\
\bar{Y}_{\left[\nu \nu^{\prime}\right] J_{k}}^{k}
\end{array}\right)=E_{k}\left(\begin{array}{c}
\bar{X}_{\left[\mu \mu^{\prime}\right] J_{k}}^{k} \\
\bar{Y}_{\left[\mu \mu^{\prime}\right] J_{k}}^{k}
\end{array}\right), \quad \mu \leq \mu^{\prime}, \\
& A_{\left[\mu \mu^{\prime}\right] J_{k},\left[\nu \nu^{\prime}\right] J_{k}}=\frac{1}{\sqrt{1+\delta_{\mu \mu^{\prime}}}} \frac{1}{\sqrt{1+\delta_{\nu \nu^{\prime}}}}\left\{E_{\mu \nu} \delta_{\mu^{\prime} \nu^{\prime}}-E_{\mu^{\prime} \nu} \delta_{\mu \nu^{\prime}}(-)^{j_{\mu}+j_{\mu^{\prime}}-J_{k}}\right. \\
& -E_{\mu \nu^{\prime}} \delta_{\mu^{\prime} \nu}(-)^{j_{\mu}+j_{\mu^{\prime}}-J_{k}}+E_{\mu^{\prime} \nu^{\prime}} \delta_{\mu \nu} \\
& +G\left(\mu \mu^{\prime} \nu \nu^{\prime} ; J_{k}\right)\left(u_{\mu^{\prime}} u_{\mu} u_{\nu} u_{\nu^{\prime}}+v_{\nu} v_{\nu^{\prime}} v_{\mu^{\prime}} v_{\mu}\right) \\
& +F\left(\mu \mu^{\prime} \nu \nu^{\prime} ; J_{k}\right)\left(u_{\mu} v_{\nu^{\prime}} u_{\nu} v_{\mu^{\prime}}+u_{\mu^{\prime}} v_{\nu} u_{\nu^{\prime}} v_{\mu}\right) \\
& -(-)^{j_{\nu^{\prime}}+j_{\nu}-J_{k}} F\left(\mu \mu^{\prime} \nu^{\prime} \nu ; J_{k}\right)\left(u_{\mu} v_{\nu} u_{\nu^{\prime}} v_{\mu^{\prime}}+u_{\mu^{\prime}} v_{\nu^{\prime}} u_{\nu} v_{\mu}\right) \\
& -H\left(\mu \mu^{\prime} \nu \nu^{\prime} ; J_{k}\right)\left(v_{\nu} v_{\nu^{\prime}} u_{\mu} v_{\mu^{\prime}}+u_{\mu^{\prime}} v_{\mu} u_{\nu} u_{\nu^{\prime}}\right) \\
& +(-)^{j_{\mu}+j_{\mu^{\prime}}-J_{k}} H\left(\mu^{\prime} \mu \nu \nu^{\prime} ; J_{k}\right)\left(v_{\nu} v_{\nu^{\prime}} u_{\mu^{\prime}} v_{\mu}+u_{\mu} v_{\mu^{\prime}} u_{\nu} u_{\nu^{\prime}}\right) \\
& -H^{*}\left(\nu \nu^{\prime} \mu \mu^{\prime} ; J_{k}\right)\left(u_{\mu} u_{\mu^{\prime}} u_{\nu^{\prime}} v_{\nu}+u_{\nu} v_{\nu^{\prime}} v_{\mu^{\prime}} v_{\mu}\right) \\
& \left.+(-)^{j_{\nu}+j_{\nu^{\prime}}-J_{k}} H^{*}\left(\nu^{\prime} \nu \mu \mu^{\prime} ; J_{k}\right)\left(u_{\mu} u_{\mu^{\prime}} u_{\nu} v_{\nu^{\prime}}+u_{\nu^{\prime}} v_{\nu} v_{\mu^{\prime}} v_{\mu}\right)\right\} \text {, } \\
& B_{\left[\mu \mu^{\prime}\right] J_{k},\left[\bar{\nu} \bar{\nu}^{\prime}\right] J_{k}}=\frac{1}{\sqrt{1+\delta_{\mu \mu^{\prime}}}} \frac{1}{\sqrt{1+\delta_{\nu \nu^{\prime}}}}\left\{-G\left(\mu \mu^{\prime} \nu \nu^{\prime} ; J_{k}\right)\left(u_{\mu^{\prime}} u_{\mu} v_{\nu} v_{\nu^{\prime}}+u_{\nu} u_{\nu^{\prime}} v_{\mu^{\prime}} v_{\mu}\right)\right. \\
& -(-)^{j_{\nu}+j_{\nu^{\prime}}-J_{k}} F\left(\mu \mu^{\prime} \nu^{\prime} \nu ; J_{k}\right)\left(u_{\mu} u_{\nu} v_{\mu^{\prime}} v_{\nu^{\prime}}+u_{\mu^{\prime}} u_{\nu^{\prime}} v_{\nu} v_{\mu}\right)
\end{aligned}
$$

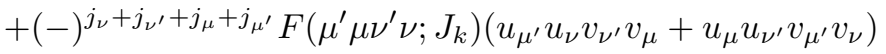

$$
\begin{aligned}
& +H\left(\mu \mu^{\prime} \nu \nu^{\prime} ; J_{k}\right)\left(v_{\nu} v_{\nu^{\prime}} u_{\mu^{\prime}} v_{\mu}+u_{\mu} v_{\mu^{\prime}} u_{\nu} u_{\nu^{\prime}}\right) \\
& -(-)^{j_{\mu}+j_{\mu^{\prime}}-J_{k}} H\left(\mu^{\prime} \mu \nu \nu^{\prime} ; J_{k}\right)\left(v_{\nu} v_{\nu^{\prime}} u_{\mu} v_{\mu^{\prime}}+u_{\mu^{\prime}} v_{\mu} u_{\nu} u_{\nu^{\prime}}\right) \\
& -H^{*}\left(\nu \nu^{\prime} \mu \mu^{\prime} ; J_{k}\right)\left(v_{\mu} v_{\mu^{\prime}} u_{\nu^{\prime}} v_{\nu}+u_{\nu} v_{\nu^{\prime}} u_{\mu} u_{\mu^{\prime}}\right) \\
& \left.+(-)^{j_{\nu}+j_{\nu^{\prime}}-J_{k}} H^{*}\left(\nu^{\prime} \nu \mu \mu^{\prime} ; J_{k}\right)\left(v_{\mu} v_{\mu^{\prime}} u_{\nu} v_{\nu^{\prime}}+u_{\nu^{\prime}} v_{\nu} u_{\mu} u_{\mu^{\prime}}\right)\right\} \text {, } \\
& G\left(\mu \mu^{\prime} \nu \nu^{\prime} ; J_{k}\right)=\sum_{m_{\mu} m_{\mu^{\prime}} m_{\nu} m_{\nu^{\prime}}}\left\langle j_{\mu} m_{\mu} j_{\mu^{\prime}} m_{\mu^{\prime}} \mid J_{k} M_{k}\right\rangle\left\langle j_{\nu} m_{\nu} j_{\nu^{\prime}} m_{\nu^{\prime}} \mid J_{k} M_{k}\right\rangle \bar{V}_{K K^{\prime} L L^{\prime}}^{\mathrm{pp}} \\
& \equiv\left\langle\left[\mu \mu^{\prime}\right] J_{k}\left|\bar{V}^{\mathrm{pp}}\right|\left[\nu \nu^{\prime}\right] J_{k}\right\rangle, \\
& F\left(\mu \mu^{\prime} \nu \nu^{\prime} ; J_{k}\right)=\sum_{m_{\mu} m_{\mu^{\prime}} m_{\nu} m_{\nu^{\prime}}}\left\langle j_{\mu} m_{\mu} j_{\mu^{\prime}} m_{\mu^{\prime}} \mid J_{k} M_{k}\right\rangle\left\langle j_{\nu} m_{\nu} j_{\nu^{\prime}} m_{\nu^{\prime}} \mid J_{k} M_{k}\right\rangle \bar{V}_{K \bar{L}^{\prime} \bar{K}^{\prime} L}^{\mathrm{ph}} \\
& =\sum_{J^{\prime}}(-)^{j_{\mu^{\prime}}+j_{\nu}+J^{\prime}}\left\{\begin{array}{lll}
j_{\mu} & j_{\mu^{\prime}} & J_{k} \\
j_{\nu} & j_{\nu^{\prime}} & J^{\prime}
\end{array}\right\}\left(2 J^{\prime}+1\right)\left\langle\left[\mu \nu^{\prime}\right] J_{k}\left|\bar{V}^{\mathrm{ph}}\right|\left[\mu^{\prime} \nu\right] J_{k}\right\rangle, \\
& H\left(\mu \mu^{\prime} \nu \nu^{\prime} ; J_{k}\right)=\sum_{m_{\mu} m_{\mu^{\prime}} m_{\nu} m_{\nu^{\prime}}}\left\langle j_{\mu} m_{\mu} j_{\mu^{\prime}} m_{\mu^{\prime}} \mid J M\right\rangle\left\langle j_{\nu} m_{\nu} j_{\nu^{\prime}} m_{\nu^{\prime}} \mid J M\right\rangle \bar{V}_{\bar{L}^{\prime} \bar{L}^{\prime} K \bar{K}^{\prime}}^{3 \mathrm{p} 1 \mathrm{~h}} \\
& =\sum_{J^{\prime}}(-)^{j_{\mu}+j_{\nu}+1+l_{\nu^{\prime}}-J_{k}-J^{\prime}}\left\{\begin{array}{ccc}
j_{\mu} & j_{\mu^{\prime}} & J_{k} \\
j_{\nu^{\prime}} & j_{\nu} & J^{\prime}
\end{array}\right\}\left(2 J^{\prime}+1\right)\left\langle[\mu \nu] J_{k}\left|\bar{V}^{3 \mathrm{p} 1 \mathrm{~h}}\right|\left[\mu^{\prime} \nu^{\prime}\right] J_{k}\right\rangle .
\end{aligned}
$$

We have represented the second derivatives of the energy functional $E\left[\rho, \kappa, \kappa^{*}\right]$ as unsymmetrized matrix elements of effective interactions $\bar{V}^{\mathrm{pp}}, \bar{V}^{\mathrm{ph}}$, and $\bar{V}^{3 \mathrm{p} 1 \mathrm{~h}}$. These effective interactions are given in App. B Although the "matrix 
elements" are unsymmetrized, the underlying two-quasiparticle states are of course antisymmetric. As a consequence, $A_{\left[\mu \mu^{\prime}\right] J_{k},\left[\nu \nu^{\prime}\right] J_{k}}=B_{\left[\mu \mu^{\prime}\right] J_{k},\left[\bar{\nu} \bar{\nu}^{\prime}\right] J_{k}}=0$ if $J_{k}$ is odd and either $\mu=\mu^{\prime}$ or $\nu=\nu^{\prime}$.

The nuclear energy functional $E\left[\rho, \kappa, \kappa^{*}\right]$ is usually separated into particle-hole (ph) and pairing pieces (again, see App. B for explicit expressions). If the pairing functional, which we will call $E_{\text {pair }}\left[\rho, \kappa, \kappa^{*}\right]$, depends on $\rho$ then the derivatives of $E_{\text {pair }}\left[\rho, \kappa, \kappa^{*}\right]$ with respect to $\rho_{K K^{\prime}}$ are called pairing-rearrangement terms [13]. In the QRPA, two kinds of pairing-rearrangement terms can arise in general. One has particle-hole character and is included in $\bar{V}_{K L K^{\prime} L^{\prime}}^{\text {ph }}$ the other affects 3-particle-1-hole $(3 \mathrm{p} 1 \mathrm{~h})$ and 1-particle-3-hole $(1 \mathrm{p} 3 \mathrm{~h})$ configurations and is represented by $\bar{V}_{K^{\prime} K L^{\prime} L}^{3 \mathrm{~h}} \mathrm{~K}^{\prime}$ and $\bar{V}_{K^{\prime} K L^{\prime} L}^{1 \mathrm{p} 3 \mathrm{~h}}$. If the $\rho$-dependence of $E_{\text {pair }}\left[\rho, \kappa, \kappa^{*}\right]$ is linear, then the ph-type pairing-rearrangement term does not appear. Furthermore, the $3 \mathrm{p} 1 \mathrm{~h}$ and $1 \mathrm{p} 3 \mathrm{~h}$ pairing-rearrangement terms arise only for $J^{\pi}=0^{+}$modes if the HFB state has $J=0$. Most existing work uses a pairing functional that is linear in $\rho$, and so needs no pairing-rearrangement terms in $J^{\pi} \neq 0^{+}$channels.

\section{APPENDIX B: INTERACTION MATRIX ELEMENTS (SECOND FUNCTIONAL DERIVATIVES)}

\section{Representation of second derivatives as matrix element of effective interactions}

In this appendix, we discuss interaction matrix elements coming from $E\left[\rho, \kappa, \kappa^{*}\right]$, which we take to contain separate Skyrme (i.e. strong-force, $\kappa$-independent), Coulomb, and pairing energy functionals:

$$
E\left[\rho, \kappa, \kappa^{*}\right]=E_{\text {Skyrme }}[\rho]+E_{\text {Coul }}\left[\rho_{\mathrm{p}}\right]+E_{\text {pair }}\left[\rho, \kappa, \kappa^{*}\right],
$$

where $\rho_{\mathrm{p}}$ is the proton density matrix. The most general Skyrme energy functional in common use is given by

$$
\begin{aligned}
E_{\text {Skyrme }}[\rho]= & \sum_{t=0,1} \int d^{3} r\left\{C_{t}^{\rho}\left[\rho_{00}\right] \rho_{t 0}^{2}(\boldsymbol{r})+C_{t}^{\Delta \rho} \rho_{t 0}(\boldsymbol{r}) \Delta \rho_{t 0}(\boldsymbol{r})+C_{t}^{\tau}\left[\rho_{t 0}(\boldsymbol{r}) \tau_{t 0}(\boldsymbol{r})-\boldsymbol{j}_{t 0}^{2}(\boldsymbol{r})\right]\right. \\
& +C_{t}^{s}\left[\rho_{00}\right] \boldsymbol{s}_{t 0}^{2}(\boldsymbol{r})+C_{t}^{\Delta s} \boldsymbol{s}_{t 0}(\boldsymbol{r}) \cdot \Delta \boldsymbol{s}_{t 0}(\boldsymbol{r})+C_{t}^{T}\left[\boldsymbol{s}_{00}(\boldsymbol{r}) \cdot \boldsymbol{T}_{t 0}(\boldsymbol{r})-\overleftrightarrow{J}_{t 0}^{2}(\boldsymbol{r})\right] \\
& \left.+C_{t}^{\nabla J}\left[\rho_{t 0}(\boldsymbol{r}) \boldsymbol{\nabla} \cdot \boldsymbol{J}_{t 0}(\boldsymbol{r})+\boldsymbol{s}_{t 0}(\boldsymbol{r}) \cdot \boldsymbol{\nabla} \times \boldsymbol{j}_{t 0}(\boldsymbol{r})\right]\right\}
\end{aligned}
$$

(See, e.g., [63, 79, 80] and references therein for a general discussion.) All densities are labeled by isospin indices $t t_{z}$, where $t$ takes values zero and one and $t_{z}$ is always equal to 0 . A more general theory could violate isospin at the single-quasiparticle level, leading to additional densities $\rho_{1 \pm 1}[63]$. We do not consider such densities here. The $C_{t}^{i}$ are the coupling constants for the effective interaction. As usual, two of them are chosen to be density dependent:

$$
\begin{aligned}
& C_{t}^{\rho}\left[\rho_{00}\right]=A_{t}^{\rho}+B_{t}^{\rho} \rho_{00}^{\alpha}(\boldsymbol{r}), \\
& C_{t}^{s}\left[\rho_{00}\right]=A_{t}^{s}+B_{t}^{s} \rho_{00}^{\alpha}(\boldsymbol{r}) .
\end{aligned}
$$

Here $\rho_{t 0}, \boldsymbol{s}_{t 0}, \tau_{t 0}, \boldsymbol{T}_{t 0}, \boldsymbol{j}_{t 0}, \overleftrightarrow{J}_{t 0}$, and $\boldsymbol{J}_{t 0}$ are local densities and currents, which are derived from the general density matrices for protons and neutrons

$$
\begin{aligned}
& \rho_{00}\left(\boldsymbol{r} \sigma, \boldsymbol{r}^{\prime} \sigma^{\prime}\right)=\rho_{\mathrm{n}}\left(\boldsymbol{r} \sigma, \boldsymbol{r}^{\prime} \sigma^{\prime}\right)+\rho_{\mathrm{p}}\left(\boldsymbol{r} \sigma, \boldsymbol{r}^{\prime} \sigma^{\prime}\right), \\
& \rho_{10}\left(\boldsymbol{r} \sigma, \boldsymbol{r}^{\prime} \sigma^{\prime}\right)=\rho_{\mathrm{n}}\left(\boldsymbol{r} \sigma, \boldsymbol{r}^{\prime} \sigma^{\prime}\right)-\rho_{\mathrm{p}}\left(\boldsymbol{r} \sigma, \boldsymbol{r}^{\prime} \sigma^{\prime}\right),
\end{aligned}
$$

where

$$
\begin{aligned}
& \rho_{\mathrm{n}}\left(\boldsymbol{r} \sigma, \boldsymbol{r}^{\prime} \sigma^{\prime}\right)=\sum_{K K^{\prime}, \text { neutron }} \psi_{K^{\prime}}^{*}\left(\boldsymbol{r}^{\prime} \sigma^{\prime}\right) \psi_{K}(\boldsymbol{r} \sigma) \rho_{K K^{\prime}}, \\
& \rho_{\mathrm{p}}\left(\boldsymbol{r} \sigma, \boldsymbol{r}^{\prime} \sigma^{\prime}\right)=\sum_{K K^{\prime}, \text { proton }} \psi_{K^{\prime}}^{*}\left(\boldsymbol{r}^{\prime} \sigma^{\prime}\right) \psi_{K}(\boldsymbol{r} \sigma) \rho_{K K^{\prime}},
\end{aligned}
$$

and $\sigma= \pm \frac{1}{2}$ labels the spin components so that, e.g., $\psi_{K}(\boldsymbol{r} \sigma)$ is a spin component of the single-particle wave function associated with the state $K$. Defining

$$
\begin{aligned}
& \rho_{t 0}\left(\boldsymbol{r}, \boldsymbol{r}^{\prime}\right)=\sum_{\sigma= \pm} \rho_{t 0}\left(\boldsymbol{r} \sigma, \boldsymbol{r}^{\prime} \sigma\right) \\
& \boldsymbol{s}_{t 0}\left(\boldsymbol{r}, \boldsymbol{r}^{\prime}\right)=\sum_{\sigma, \sigma^{\prime}= \pm} \rho_{t 0}\left(\boldsymbol{r} \sigma, \boldsymbol{r}^{\prime} \sigma^{\prime}\right) \boldsymbol{\sigma}_{\sigma^{\prime} \sigma}
\end{aligned}
$$


where $\boldsymbol{\sigma}_{\sigma^{\prime} \sigma}=\left\langle\sigma^{\prime}|\boldsymbol{\sigma}| \sigma\right\rangle$ is a matrix element of the vector of Pauli spin matrices, we write the local densities and currents as

$$
\begin{aligned}
\rho_{t 0}(\boldsymbol{r}) & =\rho_{t 0}(\boldsymbol{r}, \boldsymbol{r}) \\
\boldsymbol{s}_{t 0}(\boldsymbol{r}) & =\boldsymbol{s}_{t 0}(\boldsymbol{r}, \boldsymbol{r}) \\
\tau_{t 0}(\boldsymbol{r}) & =\left.\boldsymbol{\nabla} \cdot \boldsymbol{\nabla}^{\prime} \rho_{t 0}\left(\boldsymbol{r}, \boldsymbol{r}^{\prime}\right)\right|_{\boldsymbol{r}=\boldsymbol{r}^{\prime}} \\
\boldsymbol{T}_{t 0}(\boldsymbol{r}) & =\left.\boldsymbol{\nabla} \cdot \boldsymbol{\nabla}^{\prime} \boldsymbol{s}_{t 0}\left(\boldsymbol{r}, \boldsymbol{r}^{\prime}\right)\right|_{\boldsymbol{r}=\boldsymbol{r}^{\prime}} \\
\boldsymbol{j}_{t 0}(\boldsymbol{r}) & =-\left.\frac{i}{2}\left(\boldsymbol{\nabla}-\boldsymbol{\nabla}^{\prime}\right) \rho_{t 0}\left(\boldsymbol{r}, \boldsymbol{r}^{\prime}\right)\right|_{\boldsymbol{r}=\boldsymbol{r}^{\prime}} \\
J_{t 0, i j}(\boldsymbol{r}) & =-\left.\frac{i}{2}\left(\boldsymbol{\nabla}-\boldsymbol{\nabla}^{\prime}\right)_{i} s_{t 0, j}\left(\boldsymbol{r}, \boldsymbol{r}^{\prime}\right)\right|_{\boldsymbol{r}=\boldsymbol{r}^{\prime}} \\
\stackrel{J}{J}_{t 0}^{2}(\boldsymbol{r}) & =\sum_{i j=x y z} J_{t 0, i j}^{2}, \\
\boldsymbol{J}_{t 0}(\boldsymbol{r}) & =-\frac{i}{2}\left(\boldsymbol{\nabla}-\boldsymbol{\nabla}^{\prime}\right) \times\left.\boldsymbol{s}_{t 0}\left(\boldsymbol{r}, \boldsymbol{r}^{\prime}\right)\right|_{\boldsymbol{r}=\boldsymbol{r}^{\prime}}
\end{aligned}
$$

The Coulomb energy functional is given by

$$
E_{\text {Coul }}\left[\rho_{\mathrm{p}}\right]=\frac{e^{2}}{2} \iint d^{3} r d^{3} r^{\prime} \frac{\rho_{\mathrm{p}}(\boldsymbol{r}) \rho_{\mathrm{p}}\left(\boldsymbol{r}^{\prime}\right)}{\left|\boldsymbol{r}-\boldsymbol{r}^{\prime}\right|}-\frac{3}{4} e^{2}\left(\frac{3}{\pi}\right)^{\frac{1}{3}} \int d^{3} r \rho_{\mathrm{p}}^{4 / 3}(\boldsymbol{r})
$$

where we make the usual Slater approximation [81] for the exchange term.

For the pairing functional we take the quite general form

$$
E_{\mathrm{pair}}\left[\rho, \kappa, \kappa^{*}\right]=E_{\mathrm{pair}}\left[\rho, \tilde{\rho}, \tilde{\rho}^{*}\right]=\int d^{3} r C^{\tilde{\rho}}\left[\rho_{00}(\boldsymbol{r})\right] \sum_{\tau=\mathrm{p}, \mathrm{n}}\left|\tilde{\rho}_{\tau}(\boldsymbol{r})\right|^{2},
$$

where the density-dependent pairing coupling constant $C^{\tilde{\rho}}\left[\rho_{00}(\boldsymbol{r})\right]$ is an arbitrary function of $\rho_{00}(\boldsymbol{r})$. The quantity $\tilde{\rho}_{\tau}(\boldsymbol{r})$ is defined as 64 ]

$$
\tilde{\rho}_{\tau}(\boldsymbol{r})=-i \sum_{\sigma \sigma^{\prime}= \pm} \kappa_{\tau}\left(\boldsymbol{r} \sigma, \boldsymbol{r} \sigma^{\prime}\right) \sigma_{\sigma \sigma^{\prime}}^{y}, \tau=\text { proton or neutron, }
$$

with

$$
\begin{aligned}
& \kappa_{\mathrm{n}}\left(\boldsymbol{r} \sigma, \boldsymbol{r}^{\prime} \sigma^{\prime}\right)=\sum_{K K^{\prime}, \text { neutron }} \psi_{K^{\prime}}\left(\boldsymbol{r}^{\prime} \sigma^{\prime}\right) \psi_{K}(\boldsymbol{r} \sigma) \kappa_{K K^{\prime}}, \\
& \kappa_{\mathrm{p}}\left(\boldsymbol{r} \sigma, \boldsymbol{r}^{\prime} \sigma^{\prime}\right)=\sum_{K K^{\prime}, \text { proton }} \psi_{K^{\prime}}\left(\boldsymbol{r}^{\prime} \sigma^{\prime}\right) \psi_{K}(\boldsymbol{r} \sigma) \kappa_{K K^{\prime}},
\end{aligned}
$$

being the standard pairing tensor in the coordinate representation.

The second derivatives of the energy functional in Eq. (A4), as the equation indicates and we've already noted, can be written as unsymmetrized matrix elements $\bar{V}_{K L K^{\prime} L^{\prime}}^{\mathrm{ph}}$ of an effective interaction between uncoupled pairs of single-particle states. The particle-hole matrix elements take the form

$$
\bar{V}_{K L K^{\prime} L^{\prime}}^{\mathrm{ph}}=\left\langle K L\left|\bar{V}_{\text {Skyrme }}^{\text {eff }}+\bar{V}_{\text {Coul }}^{\text {eff }}+\bar{V}_{\text {pair }}^{\text {eff }}{ }^{\text {ph }}\right| K^{\prime} L^{\prime}\right\rangle .
$$

The last term contains the pairing rearrangement discussed at the end of the previous appendix.

The effective Skyrme interaction in Eq. (B12) is given by

$$
\begin{aligned}
\bar{V}_{\text {Skyrme }}^{\mathrm{eff}}= & \left(a_{0}+b_{0} \boldsymbol{\sigma} \cdot \boldsymbol{\sigma}^{\prime}+c_{0} \vec{\tau} \cdot \vec{\tau}^{\prime}+d_{0} \boldsymbol{\sigma} \cdot \boldsymbol{\sigma}^{\prime} \vec{\tau} \cdot \vec{\tau}^{\prime}\right) \delta\left(\boldsymbol{r}-\boldsymbol{r}^{\prime}\right) \\
& +\left(a_{1}+b_{1} \boldsymbol{\sigma} \cdot \boldsymbol{\sigma}^{\prime}+c_{1} \vec{\tau} \cdot \vec{\tau}^{\prime}+d_{1} \boldsymbol{\sigma} \cdot \boldsymbol{\sigma}^{\prime} \vec{\tau} \cdot \vec{\tau}^{\prime}\right)\left(\boldsymbol{k}^{\dagger} \delta\left(\boldsymbol{r}-\boldsymbol{r}^{\prime}\right)+\delta\left(\boldsymbol{r}-\boldsymbol{r}^{\prime}\right) \boldsymbol{k}^{2}\right) \\
& +\left(a_{2}+b_{2} \boldsymbol{\sigma} \cdot \boldsymbol{\sigma}^{\prime}+c_{2} \vec{\tau} \cdot \vec{\tau}^{\prime}+d_{2} \boldsymbol{\sigma} \cdot \boldsymbol{\sigma}^{\prime} \vec{\tau} \cdot \vec{\tau}^{\prime}\right) \boldsymbol{k}^{\dagger} \cdot \delta\left(\boldsymbol{r}-\boldsymbol{r}^{\prime}\right) \boldsymbol{k} \\
& +\left(a_{3}+b_{3} \boldsymbol{\sigma} \cdot \boldsymbol{\sigma}^{\prime}+c_{3} \vec{\tau} \cdot \vec{\tau}^{\prime}+d_{3} \boldsymbol{\sigma} \cdot \boldsymbol{\sigma}^{\prime} \vec{\tau} \cdot \vec{\tau}^{\prime}\right) \rho_{00}^{\alpha}(\boldsymbol{r}) \delta\left(\boldsymbol{r}-\boldsymbol{r}^{\prime}\right) \\
& +\left[e_{3} \rho_{10}(\boldsymbol{r})\left(\tau^{(0)}+\tau^{\prime(0)}\right)+g_{3} \boldsymbol{s}_{00}(\boldsymbol{r}) \cdot\left(\boldsymbol{\sigma}+\boldsymbol{\sigma}^{\prime}\right)\right. \\
& \left.\quad+m_{3} \boldsymbol{s}_{10}(\boldsymbol{r}) \cdot\left(\boldsymbol{\sigma} \tau^{(0)}+\boldsymbol{\sigma}^{\prime} \tau^{\prime(0)}\right)\right] \rho_{00}^{\alpha-1}(\boldsymbol{r}) \delta\left(\boldsymbol{r}-\boldsymbol{r}^{\prime}\right) \\
& +\left[f_{3} \rho_{10}^{2}(\boldsymbol{r})+h_{3} \boldsymbol{s}_{00}^{2}(\boldsymbol{r})+n_{3} \boldsymbol{s}_{10}^{2}(\boldsymbol{r})\right] \rho_{00}^{\alpha-2}(\boldsymbol{r}) \delta\left(\boldsymbol{r}-\boldsymbol{r}^{\prime}\right) \\
& +\left(a_{4}+c_{4} \vec{\tau} \cdot \vec{\tau}^{\prime}\right)\left(\boldsymbol{\sigma}+\boldsymbol{\sigma}^{\prime}\right) \cdot \boldsymbol{k}^{\dagger} \times \delta\left(\boldsymbol{r}-\boldsymbol{r}^{\prime}\right) \boldsymbol{k} .
\end{aligned}
$$


TABLE III: Definitions of $a_{i}, b_{i}, c_{i}, d_{i}(i=0, \cdots, 3), a_{4}$, and $c_{4}$ in Eq. (B13).

\begin{tabular}{lcccc}
\hline \hline$i$ & $a_{i}$ & $b_{i}$ & $c_{i}$ & $d_{i}$ \\
\hline 0 & $2 A_{0}^{\rho}$ & $2 A_{0}^{s}$ & $2 A_{1}^{\rho}$ & $2 A_{1}^{s}$ \\
1 & $\frac{1}{2}\left(C_{0}^{\tau}-4 C_{0}^{\Delta \rho}\right)$ & $\frac{1}{2}\left(C_{0}^{T}-4 C_{0}^{\Delta s}\right)$ & $\frac{1}{2}\left(C_{1}^{\tau}-4 C_{1}^{\Delta \rho}\right)$ & $\frac{1}{2}\left(C_{1}^{T}-4 C_{1}^{\Delta s}\right)$ \\
2 & $3 C_{0}^{\tau}+4 C_{0}^{\Delta \rho}$ & $3 C_{0}^{T}+4 C_{0}^{\Delta s}$ & $3 C_{1}^{\tau}+4 C_{1}^{\Delta \rho}$ & $3 C_{1}^{T}+4 C_{1}^{\Delta s}$ \\
3 & $B_{0}^{\rho}(\alpha+2)(\alpha+1)$ & $2 B_{0}^{s}$ & $2 B_{1}^{\rho}$ & $2 B_{1}^{s}$ \\
4 & $-2 i C_{0}^{\nabla J}$ & & $-2 i C_{1}^{\nabla J}$ & \\
\hline \hline
\end{tabular}

TABLE IV: Definitions of the coefficients appearing in the rearrangement terms in Eq. (B13).

\begin{tabular}{ccccccc}
\hline \hline$i$ & $e_{i}$ & $f_{i}$ & $g_{i}$ & $h_{i}$ & $m_{i}$ & $n_{i}$ \\
\hline 3 & $2 \alpha B_{1}^{\rho}$ & $\alpha(\alpha-1) B_{1}^{\rho}$ & $2 \alpha B_{0}^{s}$ & $\alpha(\alpha-1) B_{0}^{s}$ & $2 \alpha B_{1}^{s}$ & $\alpha(\alpha-1) B_{1}^{s}$ \\
\hline \hline
\end{tabular}

$\vec{\tau}=\left(\tau^{( \pm 1)}, \tau^{(0)}\right)$ is the vector of Pauli matrices in isospin space and

$$
\begin{aligned}
\boldsymbol{k}=-\frac{i}{2}\left(\boldsymbol{\nabla}-\boldsymbol{\nabla}^{\prime}\right) & \text { acting to the right, } \\
\boldsymbol{k}^{\dagger}=\frac{i}{2}\left(\boldsymbol{\nabla}-\boldsymbol{\nabla}^{\prime}\right) & \text { acting to the left. }
\end{aligned}
$$

The coefficients in Eq. (B13) are defined in Tables III and IV Equation (B13) contains the usual Skyrme-interaction operators, but, the energy functional (B2) does not necessarily correspond to a real (density-dependent) two-body Skyrme interaction because the matrix elements are not antisymmetrized. Compared to the case usually discussed in the literature, the more general functional relaxes relations that would otherwise restrict the spin-isospin structure of the effective interaction in Eq. (B13); see, e.g., [16] for a discussion of the increased freedom.

The densities and currents that appear in Eq. (B13) come mostly from rearrangement terms and take the values given by the HFB ground state. The isoscalar and isovector spin densities $\boldsymbol{s}_{t 0}(\boldsymbol{r})$ vanish when the HFB ground state is time-reversal invariant or spherical as assumed here. The terms containing them will therefore not appear in the expressions for the matrix elements of the effective interaction for such states given below.

The effective Coulomb interaction in Eq. (B12), acting between protons, is given by

$$
\bar{V}_{\mathrm{Coul}}^{\mathrm{eff}}=\frac{e^{2}}{\left|\boldsymbol{r}-\boldsymbol{r}^{\prime}\right|}-\frac{e^{2}}{3}\left(\frac{3}{\pi}\right)^{\frac{1}{3}} \rho_{\mathrm{p}}^{-2 / 3}(\boldsymbol{r}) \delta\left(\boldsymbol{r}-\boldsymbol{r}^{\prime}\right)
$$

Finally, the ph-type pairing-rearrangement terms in Eq. (B12) come from an effective interaction

$$
\bar{V}_{\text {pair }}^{\text {eff ph }}=\frac{d^{2} C^{\tilde{\rho}}\left[\rho_{00}(\boldsymbol{r})\right]}{d \rho_{00}^{2}(\boldsymbol{r})} \sum_{\tau=\mathrm{p}, \mathrm{n}}\left|\tilde{\rho}_{\tau}(\boldsymbol{r})\right|^{2} \delta\left(\boldsymbol{r}^{\prime}-\boldsymbol{r}\right)
$$

The second derivatives with respect to $\kappa, \kappa^{*}$ also can be written as unsymmetrized matrix elements of effective interactions, this time in the particle-particle channel. The particle-particle effective interaction entering the matrix elements

$$
\bar{V}_{K K^{\prime} L L^{\prime}}^{\mathrm{pp}}=\left\langle K K^{\prime}\left|\bar{V}_{\mathrm{pair}}^{\mathrm{eff} p \mathrm{pp}}\right| L L^{\prime}\right\rangle
$$

is obtained from Eq. (B9) through Eq. A5 as

$$
\bar{V}_{\text {pair }}^{\text {eff pp }}=C^{\tilde{\rho}}\left[\rho_{00}(\boldsymbol{r})\right]\left(3-\boldsymbol{\sigma} \cdot \boldsymbol{\sigma}^{\prime}-\vec{\tau} \cdot \vec{\tau}^{\prime}-\boldsymbol{\sigma} \cdot \boldsymbol{\sigma}^{\prime} \vec{\tau} \cdot \vec{\tau}^{\prime}\right) \delta\left(\boldsymbol{r}-\boldsymbol{r}^{\prime}\right) .
$$

In the numerical calculations of this paper, we use a volume pairing-energy functional, i.e.,

$$
C^{\tilde{\rho}}=\frac{1}{2} V_{0}=\text { const. }
$$

Last of all are the mixed functional derivatives involving both $\rho$ and $\kappa$ (or $\tilde{\rho}$ ) in Eq. (A6). They also can be written as the unsymmetrized matrix elements of an effective interaction:

$$
\bar{V}_{K^{\prime} K L^{\prime} L}^{3 \mathrm{p} 1 \mathrm{~h}}=\left\langle L^{\prime} K^{\prime}\left|\bar{V}_{\text {pair }}^{\text {eff } 3 \mathrm{p} 1 \mathrm{~h}}\right| L T(K)\right\rangle,
$$


where $T(K)$ denotes the time-reversed state of $K$, and the $3 p 1 h$ effective interaction itself is

$$
\bar{V}_{\text {pair }}^{\text {eff } 3 \mathrm{p} 1 \mathrm{~h}}=\frac{d C^{\tilde{\rho}}\left[\rho_{00}(\boldsymbol{r})\right]}{d \rho_{00}(\boldsymbol{r})}\left[\tilde{\rho}_{\mathrm{p}}(\boldsymbol{r})\left(1-\tau_{z}^{\prime}\right)+\tilde{\rho}_{\mathrm{n}}(\boldsymbol{r})\left(1+\tau_{z}^{\prime}\right)\right] \delta\left(\boldsymbol{r}^{\prime}-\boldsymbol{r}\right),
$$

where $\tau_{z}^{\prime}$ acts on the single-particle states $K^{\prime}$ and $T(K)$ in Eq. (B20), and the eigenvalues 1 and -1 are assigned to the neutron and proton, respectively.

\section{Calculation of matrix elements}

To calculate the coupled matrix elements in Eqs. A14 A16, we use an intermediate LS scheme:

$$
\begin{gathered}
\left\langle\left[\mu \mu^{\prime}\right] J_{k}|\bar{V}|\left[\nu \nu^{\prime}\right] J_{k}\right\rangle \\
=\sum_{L L^{\prime} S} \hat{j}_{\mu} \hat{j}_{\mu^{\prime}} \hat{j}_{\nu} \hat{j}_{\nu^{\prime}} \hat{L} \hat{L}^{\prime} \hat{S}^{2}\left\{\begin{array}{ccc}
l_{\mu} & l_{\mu^{\prime}} & L \\
1 / 2 & 1 / 2 & S \\
j_{\mu} & j_{\mu^{\prime}} & J_{k}
\end{array}\right\}\left\{\begin{array}{ccc}
l_{\nu} & l_{\nu^{\prime}} & L^{\prime} \\
1 / 2 & 1 / 2 & S \\
j_{\nu} & j_{\nu^{\prime}} & J_{k}
\end{array}\right\} \\
\quad \times\left\langle\left(l_{\mu} l_{\mu^{\prime}}\right) L S ; J_{k}|\bar{V}|\left(l_{\nu} l_{\nu^{\prime}}\right) L^{\prime} S ; J_{k}\right\rangle, \\
\hat{j}_{\mu} \equiv \sqrt{2 j_{\mu}+1} .
\end{gathered}
$$

Eq. (B13) gives

i) proton-proton or neutron-neutron matrix elements:

$$
\begin{aligned}
& \left\langle\left(l_{\mu} l_{\mu^{\prime}}\right) L S ; J_{k}\left|\bar{V}_{\text {Skyrme }}^{\text {eff }}\right|\left(l_{\nu} l_{\nu^{\prime}}\right) L^{\prime} S ; J_{k}\right\rangle \\
& =\left\{a_{0}+c_{0}+(2 S(S+1)-3)\left(b_{0}+d_{0}\right)\right\}\left\langle\left(l_{\mu} l_{\mu^{\prime}}\right) L S ; J_{k}\left|\delta\left(\boldsymbol{r}-\boldsymbol{r}^{\prime}\right)\right|\left(l_{\nu} l_{\nu^{\prime}}\right) L^{\prime} S ; J_{k}\right\rangle \\
& +\left\{a_{1}+c_{1}+(2 S(S+1)-3)\left(b_{1}+d_{1}\right)\right\}\left\langle\left(l_{\mu} l_{\mu^{\prime}}\right) L S ; J_{k}\left|\boldsymbol{k}^{\dagger 2} \delta\left(\boldsymbol{r}-\boldsymbol{r}^{\prime}\right)+\delta\left(\boldsymbol{r}-\boldsymbol{r}^{\prime}\right) \boldsymbol{k}^{2}\right|\left(l_{\nu} l_{\nu^{\prime}}\right) L^{\prime} S ; J_{k}\right\rangle \\
& +\left\{a_{2}+c_{2}+(2 S(S+1)-3)\left(b_{2}+d_{2}\right)\right\}\left\langle\left(l_{\mu} l_{\mu^{\prime}}\right) L S ; J_{k}\left|\boldsymbol{k}^{\dagger} \cdot \delta\left(\boldsymbol{r}-\boldsymbol{r}^{\prime}\right) \boldsymbol{k}\right|\left(l_{\nu} l_{\nu^{\prime}}\right) L^{\prime} S ; J_{k}\right\rangle \\
& +\left\{a_{3}+c_{3}+(2 S(S+1)-3)\left(b_{3}+d_{3}\right)\right\}\left\langle\left(l_{\mu} l_{\mu^{\prime}}\right) L S ; J_{k}\left|\rho_{00}^{\alpha}(\boldsymbol{r}) \delta\left(\boldsymbol{r}-\boldsymbol{r}^{\prime}\right)\right|\left(l_{\nu} l_{\nu^{\prime}}\right) L^{\prime} S ; J_{k}\right\rangle \\
& +2 e_{3}\left\langle\left(l_{\mu} l_{\mu^{\prime}}\right) L S ; J_{k}\left|\rho_{10}(\boldsymbol{r}) \rho_{00}^{\alpha-1}(\boldsymbol{r}) \delta\left(\boldsymbol{r}-\boldsymbol{r}^{\prime}\right)\right|\left(l_{\nu} l_{\nu^{\prime}}\right) L^{\prime} S ; J_{k}\right\rangle \times\left\{\begin{array}{cc}
(-1), & \text { proton } \\
1, & \text { neutron }
\end{array}\right. \\
& +f_{3}\left\langle\left(l_{\mu} l_{\mu^{\prime}}\right) L S ; J_{k}\left|\rho_{10}^{2}(\boldsymbol{r}) \rho_{00}^{\alpha-2}(\boldsymbol{r}) \delta\left(\boldsymbol{r}-\boldsymbol{r}^{\prime}\right)\right|\left(l_{\nu} l_{\nu^{\prime}}\right) L^{\prime} S ; J_{k}\right\rangle \\
& +\left(a_{4}+c_{4}\right)\left\langle\left(l_{\mu} l_{\mu^{\prime}}\right) L S ; J_{k}\left|\left(\boldsymbol{\sigma}+\boldsymbol{\sigma}^{\prime}\right) \cdot \boldsymbol{k}^{\dagger} \times \delta\left(\boldsymbol{r}-\boldsymbol{r}^{\prime}\right) \boldsymbol{k}\right|\left(l_{\nu} l_{\nu^{\prime}}\right) L^{\prime} S ; J_{k}\right\rangle \text {, }
\end{aligned}
$$

ii) proton-neutron matrix elements:

$$
\begin{aligned}
\left\langle\left(l_{\mu} l_{\mu^{\prime}}\right) L S ; J_{k}\left|\bar{V}_{\mathrm{Skyrme}}^{\mathrm{eff}}\right|\left(l_{\nu} l_{\nu^{\prime}}\right) L^{\prime} S ; J_{k}\right\rangle \\
=\quad\left\{a_{0}-c_{0}+(2 S(S+1)-3)\left(b_{0}-d_{0}\right)\right\}\left\langle\left(l_{\mu} l_{\mu^{\prime}}\right) L S ; J_{k}\left|\delta\left(\boldsymbol{r}-\boldsymbol{r}^{\prime}\right)\right|\left(l_{\nu} l_{\nu^{\prime}}\right) L^{\prime} S ; J_{k}\right\rangle \\
\quad+\left\{a_{1}-c_{1}+(2 S(S+1)-3)\left(b_{1}-d_{1}\right)\right\}\left\langle\left(l_{\mu} l_{\mu^{\prime}}\right) L S ; J_{k}\left|\boldsymbol{k}^{\dagger} \delta\left(\boldsymbol{r}-\boldsymbol{r}^{\prime}\right)+\delta\left(\boldsymbol{r}-\boldsymbol{r}^{\prime}\right) \boldsymbol{k}^{2}\right|\left(l_{\nu} l_{\nu^{\prime}}\right) L^{\prime} S ; J_{k}\right\rangle \\
\quad+\left\{a_{2}-c_{2}+(2 S(S+1)-3)\left(b_{2}-d_{2}\right)\right\}\left\langle\left(l_{\mu} l_{\mu^{\prime}}\right) L S ; J_{k}\left|\boldsymbol{k}^{\dagger} \cdot \delta\left(\boldsymbol{r}-\boldsymbol{r}^{\prime}\right) \boldsymbol{k}\right|\left(l_{\nu} l_{\nu^{\prime}}\right) L^{\prime} S ; J_{k}\right\rangle \\
\quad+\left\{a_{3}-c_{3}+(2 S(S+1)-3)\left(b_{3}-d_{3}\right)\right\}\left\langle\left(l_{\mu} l_{\mu^{\prime}}\right) L S ; J_{k}\left|\rho_{00}^{\alpha}(\boldsymbol{r}) \delta\left(\boldsymbol{r}-\boldsymbol{r}^{\prime}\right)\right|\left(l_{\nu} l_{\nu^{\prime}}\right) L^{\prime} S ; J_{k}\right\rangle \\
\quad+f_{3}\left\langle\left(l_{\mu} l_{\mu^{\prime}}\right) L S ; J_{k}\left|\rho_{10}^{2}(\boldsymbol{r}) \rho_{00}^{\alpha-2}(\boldsymbol{r}) \delta\left(\boldsymbol{r}-\boldsymbol{r}^{\prime}\right)\right|\left(l_{\nu} l_{\nu^{\prime}}\right) L^{\prime} S ; J_{k}\right\rangle \\
\quad+\left(a_{4}-c_{4}\right)\left\langle\left(l_{\mu} l_{\mu^{\prime}}\right) L S ; J_{k}\left|\left(\boldsymbol{\sigma}+\boldsymbol{\sigma}^{\prime}\right) \cdot \boldsymbol{k}^{\dagger} \times \delta\left(\boldsymbol{r}-\boldsymbol{r}^{\prime}\right) \boldsymbol{k}\right|\left(l_{\nu} l_{\nu^{\prime}}\right) L^{\prime} S ; J_{k}\right\rangle .
\end{aligned}
$$

We use the canonical (and real) radial wave functions $R_{\mu}(r)$, the angular wave functions $Y_{l_{\mu}} l_{\mu}^{z}(\Omega)$, and the spin wave functions to write the nontrivial matrix elements included in Eqs. (B24) and (B25) as

$$
\begin{aligned}
& \left\langle\left(l_{\mu} l_{\mu^{\prime}}\right) L S ; J_{k}\left|\delta\left(\boldsymbol{r}-\boldsymbol{r}^{\prime}\right)\right|\left(l_{\nu} l_{\nu^{\prime}}\right) L^{\prime} S ; J_{k}\right\rangle \\
& =\int d r r^{2} R_{\mu}(r) R_{\mu^{\prime}}(r) R_{\nu}(r) R_{\nu^{\prime}}(r) \delta_{L L^{\prime}} \int d \Omega\left[Y_{l_{\mu}}(\Omega) Y_{l_{\mu^{\prime}}}(\Omega)\right]_{L 0}^{*}\left[Y_{l_{\nu}}(\Omega) Y_{l_{\nu^{\prime}}}(\Omega)\right]_{L 0},
\end{aligned}
$$




$$
\begin{aligned}
& \int d \Omega\left[Y_{l_{\mu}}(\Omega) Y_{l_{\mu^{\prime}}}(\Omega)\right]_{L 0}^{*}\left[Y_{l_{\nu}}(\Omega) Y_{l_{\nu^{\prime}}}(\Omega)\right]_{L 0}=\frac{1}{4 \pi} \hat{l_{\mu}} \hat{l_{\mu^{\prime}}} \hat{l_{\nu}} \hat{l_{\nu^{\prime}}}\left(\begin{array}{ccc}
l_{\mu} & l_{\mu^{\prime}} & L \\
0 & 0 & 0
\end{array}\right)\left(\begin{array}{ccc}
l_{\nu} & l_{\nu^{\prime}} & L \\
0 & 0 & 0
\end{array}\right), \\
& \left\langle\left(l_{\mu} l_{\mu^{\prime}}\right) L S ; J_{k}\left|\delta\left(\boldsymbol{r}-\boldsymbol{r}^{\prime}\right) \boldsymbol{k}^{2}\right|\left(l_{\nu} l_{\nu^{\prime}}\right) L^{\prime} S ; J_{k}\right\rangle \\
& =-\frac{1}{4} \delta_{L L^{\prime}} \int d \Omega\left[Y_{l_{\mu}}(\Omega) Y_{l_{\mu^{\prime}}}(\Omega)\right]_{L 0}^{*}\left[Y_{l_{\nu}}(\Omega) Y_{l_{\nu^{\prime}}}(\Omega)\right]_{L 0} \\
& \times \int d r r^{2} R_{\mu}(r) R_{\mu^{\prime}}(r)\left\{\left[\left(\frac{d^{2}}{d r^{2}}+\frac{2}{r} \frac{d}{d r}-\frac{l_{\nu}\left(l_{\nu}+1\right)}{r^{2}}\right) R_{\nu}(r)\right] R_{\nu^{\prime}}(r)\right. \\
& \left.+R_{\nu}(r)\left[\left(\frac{d^{2}}{d r^{2}}+\frac{2}{r} \frac{d}{d r}-\frac{l_{\nu^{\prime}}\left(l_{\nu^{\prime}}+1\right)}{r^{2}}\right) R_{\nu^{\prime}}(r)\right]\right\} \\
& -\sum_{\Delta l_{\nu}=0,1} \sum_{\Delta l_{\nu^{\prime}}=0,1} \frac{1}{2} \sqrt{\left(l_{\nu}+\Delta l_{\nu}\right)\left(l_{\nu^{\prime}}+\Delta l_{\nu^{\prime}}\right)}\left\{\begin{array}{cc}
l_{\nu^{\prime}}-1+2 \Delta l_{\nu^{\prime}} & l_{\nu}-1+2 \Delta l_{\nu} \\
l_{\nu} & L \\
l_{\nu^{\prime}} & 1
\end{array}\right\} \\
& \times \int d r r^{2} R_{\mu}(r) R_{\mu^{\prime}}(r)\left\{\left(l_{\nu}+1-\Delta l_{\nu}\right) \frac{R_{\nu}(r)}{r}+(-)^{\Delta l_{\nu}} \frac{d R_{\nu}(r)}{d r}\right\} \\
& \times\left\{\left(l_{\nu^{\prime}}+1-\Delta l_{\nu^{\prime}}\right) \frac{R_{\nu^{\prime}}(r)}{r}+(-)^{\Delta l_{\nu^{\prime}}} \frac{d R_{\nu^{\prime}}(r)}{d r}\right\} \\
& \times \delta_{L L^{\prime}} \int d \Omega\left[Y_{l_{\mu}}(\Omega) Y_{l_{\mu^{\prime}}}(\Omega)\right]_{L 0}^{*}\left[Y_{l_{\nu^{\prime}}-1+2 \Delta l_{\nu^{\prime}}}(\Omega) Y_{l_{\nu}-1+2 \Delta l_{\nu}}(\Omega)\right]_{L 0}, \\
& \left\langle\left(l_{\mu} l_{\mu^{\prime}}\right) L S ; J_{k}\left|\boldsymbol{k}^{\dagger} \cdot \delta\left(\boldsymbol{r}-\boldsymbol{r}^{\prime}\right) \boldsymbol{k}\right|\left(l_{\nu} l_{\nu^{\prime}}\right) L^{\prime} S ; J_{k}\right\rangle=-\frac{\sqrt{3}}{\hat{L}} \delta_{L L^{\prime}}\left\langle\left(l_{\mu} l_{\mu^{\prime}}\right) L\left\|\left(\boldsymbol{k}^{\dagger} \cdot \delta\left(\boldsymbol{r}-\boldsymbol{r}^{\prime}\right) \boldsymbol{k}\right)_{0}\right\|\left(l_{\nu} l_{\nu^{\prime}}\right) L\right\rangle \\
& \left\langle\left(l_{\mu} l_{\mu^{\prime}}\right) L S ; J_{k}\left|i\left(\boldsymbol{\sigma}+\boldsymbol{\sigma}^{\prime}\right) \cdot \boldsymbol{k}^{\dagger} \times \delta\left(\boldsymbol{r}-\boldsymbol{r}^{\prime}\right) \boldsymbol{k}\right|\left(l_{\nu} l_{\nu^{\prime}}\right) L^{\prime} S ; J_{k}\right\rangle \\
& =(-)^{1+L^{\prime}+J_{k}} 4 \sqrt{3}\left\{\begin{array}{ccc}
1 & L^{\prime} & J_{k} \\
L & 1 & 1
\end{array}\right\} \delta_{S 1}\left\langle\left(l_{\mu} l_{\mu^{\prime}}\right) L\left\|\left(\boldsymbol{k}^{\dagger} \cdot \delta\left(\boldsymbol{r}-\boldsymbol{r}^{\prime}\right) \boldsymbol{k}\right)_{1}\right\|\left(l_{\nu} l_{\nu^{\prime}}\right) L^{\prime}\right\rangle \text {. }
\end{aligned}
$$

The square brackets around products of spherical harmonics and the parentheses surrounding products of operators indicate angular-momentum coupling.

To evaluate Eqs. (B29) and (B30), one can use

$$
\begin{aligned}
& \left\langle\left(l_{\mu} l_{\mu^{\prime}}\right) L\left\|\left(\boldsymbol{k}^{\dagger} \cdot \delta\left(\boldsymbol{r}-\boldsymbol{r}^{\prime}\right) \boldsymbol{k}\right)_{I}\right\|\left(l_{\nu} l_{\nu^{\prime}}\right) L^{\prime}\right\rangle \\
& =\left[\frac{1}{4} \sum_{\Delta l_{\mu}=0,1} \sum_{\Delta l_{\nu}=0,1} \sum_{l_{\mu \mu^{\prime}}} \int d r r^{2}\left\{\left(l_{\mu}+1-\Delta l_{\mu}\right) \frac{R_{\mu}(r)}{r}+(-)^{\Delta l_{\mu}} \frac{d R_{\mu}(r)}{d r}\right\} R_{\mu^{\prime}}(r)\right. \\
& \times\left\{\left(l_{\nu}+1-\Delta l_{\nu}\right) \frac{R_{\nu}(r)}{r}+(-)^{\Delta l_{\nu}} \frac{d R_{\nu}(r)}{d r}\right\} R_{\nu^{\prime}}(r) \\
& \times \sqrt{l_{\mu}+\Delta l_{\mu}} \sqrt{l_{\nu}+\Delta l_{\nu}} \sqrt{2 l_{\mu}+4 \Delta l_{\mu}-1} \hat{l}_{\mu^{\prime}} \sqrt{2 l_{\nu}+4 \Delta l_{\nu}-1} \hat{l_{\nu^{\prime}}} \\
& \times \frac{1}{4 \pi} \hat{l}_{\mu \mu^{\prime}}^{2} \hat{L} \hat{L}^{\prime} \hat{I}(-)^{l_{\mu}+l_{\mu^{\prime}}+L+I+1}\left\{\begin{array}{ccc}
I & L^{\prime} & L \\
l_{\mu \mu^{\prime}} & 1 & 1
\end{array}\right\}\left\{\begin{array}{ccc}
l_{\mu \mu^{\prime}} & L & 1 \\
l_{\mu} & l_{\mu}+2 \Delta l_{\mu}-1 & l_{\mu^{\prime}}
\end{array}\right\} \\
& \times\left\{\begin{array}{ccc}
l_{\mu \mu^{\prime}} & L^{\prime} & 1 \\
l_{\nu} & l_{\nu}+2 \Delta l_{\nu}-1 & l_{\nu^{\prime}}
\end{array}\right\}\left(\begin{array}{ccc}
l_{\mu}+2 \Delta l_{\mu}-1 & l_{\mu^{\prime}} & l_{\mu \mu^{\prime}} \\
0 & 0 & 0
\end{array}\right) \\
& \left.\times\left(\begin{array}{ccc}
l_{\nu}+2 \Delta l_{\nu}-1 & l_{\nu^{\prime}} & l_{\mu \mu^{\prime}} \\
0 & 0 & 0
\end{array}\right)\right]-(-)^{l_{\nu}+l_{\nu^{\prime}}+L^{\prime}}\left[\nu \leftrightarrow \nu^{\prime}\right]-(-)^{l_{\mu}+l_{\mu^{\prime}}+L}\left[\mu \leftrightarrow \mu^{\prime}\right] \\
& +\left[\mu \leftrightarrow \mu^{\prime} \text { and } \nu \leftrightarrow \nu^{\prime}\right]
\end{aligned}
$$

where for reduced matrix elements we have used the convention

$$
\left\langle L L_{z}\left|\hat{O}_{l m}\right| L^{\prime} L_{z}^{\prime}\right\rangle=\frac{1}{\hat{L}}\left\langle L^{\prime} L_{z}^{\prime} \operatorname{lm} \mid L L_{z}\right\rangle\left\langle L|| \hat{O}_{l}|| L^{\prime}\right\rangle,
$$


and made the abbreviation

$$
\begin{aligned}
& {\left[A_{\mu \mu^{\prime} \nu \nu^{\prime}}\right]-(-)^{l_{\nu}+l_{\nu^{\prime}}+L^{\prime}}\left[\nu \leftrightarrow \nu^{\prime}\right]-(-)^{l_{\mu}+l_{\mu^{\prime}}+L}\left[\mu \leftrightarrow \mu^{\prime}\right]+\left[\mu \leftrightarrow \mu^{\prime} \text { and } \nu \leftrightarrow \nu^{\prime}\right] } \\
\equiv & A_{\mu \mu^{\prime} \nu \nu^{\prime}}-(-)^{l_{\nu}+l_{\nu^{\prime}}+L^{\prime}} A_{\mu \mu^{\prime} \nu^{\prime} \nu}-(-)^{l_{\mu}+l_{\mu^{\prime}}+L} A_{\mu^{\prime} \mu \nu \nu^{\prime}}+A_{\mu^{\prime} \mu \nu^{\prime} \nu} .
\end{aligned}
$$

Eq. (B26), modified to include additional factors in the radial integral, can also be used (together with the subsequent equations) to evaluate the matrix elements of the terms involving $\rho_{00}^{\alpha}(\boldsymbol{r})$ in $\bar{V}_{\text {Skyrme }}^{\text {eff }}$, the Coulomb-exchange interaction, and the contributions of the pairing functional to the effective ph, pp, and $3 \mathrm{p} 1 \mathrm{~h}$ interactions. The Coulomb-direct term can be evaluated in a similar but slightly more complicated way, via a multipole expansion.

In the main part of this paper we used the Skyrme functional $\mathrm{SkM}^{*}$, which is usually parameterized as in interaction in terms of coefficients $t_{0}, t_{1}, t_{2}, t_{3}, x_{0}, x_{1}, x_{2}, x_{3}$, and $W_{0}$. The relations between these coefficients and those used here, if no terms are neglected, are [63, 79]

$$
\begin{array}{ll}
C_{0}^{\rho}=\frac{3}{8} t_{0}+\frac{3}{48} t_{3} \rho_{00}^{\alpha}, & C_{1}^{\rho}=-\frac{1}{4} t_{0}\left(\frac{1}{2}+x_{0}\right)-\frac{1}{24} t_{3}\left(\frac{1}{2}+x_{3}\right) \rho_{00}^{\alpha}, \\
C_{0}^{s}=-\frac{1}{4} t_{0}\left(\frac{1}{2}-x_{0}\right)-\frac{1}{24} t_{3}\left(\frac{1}{2}-x_{3}\right) \rho_{00}^{\alpha}, & C_{1}^{s}=-\frac{1}{8} t_{0}-\frac{1}{48} t_{3} \rho_{00}^{\alpha}, \\
C_{0}^{\tau}=\frac{3}{16} t_{1}+\frac{1}{4} t_{2}\left(\frac{5}{4}+x_{2}\right), & C_{1}^{\tau}=-\frac{1}{8} t_{1}\left(\frac{1}{2}+x_{1}\right)+\frac{1}{8} t_{2}\left(\frac{1}{2}+x_{2}\right), \\
C_{0}^{T}=-\frac{1}{8} t_{1}\left(\frac{1}{2}-x_{1}\right)+\frac{1}{8} t_{2}\left(\frac{1}{2}+x_{2}\right), & C_{1}^{T}=-\frac{1}{16} t_{1}+\frac{1}{16} t_{2}, \\
C_{0}^{\Delta \rho}=-\frac{9}{64} t_{1}+\frac{1}{16} t_{2}\left(\frac{5}{4}+x_{2}\right), & C_{1}^{\Delta \rho}=\frac{3}{32} t_{1}\left(\frac{1}{2}+x_{1}\right)+\frac{1}{32} t_{2}\left(\frac{1}{2}+x_{2}\right), \\
C_{0}^{\Delta s}=\frac{3}{32} t_{1}\left(\frac{1}{2}-x_{1}\right)+\frac{1}{32} t_{2}\left(\frac{1}{2}+x_{2}\right), & C_{1}^{\Delta s}=\frac{3}{64} t_{1}+\frac{1}{64} t_{2}, \\
C_{0}^{\nabla J}=-\frac{3}{4} W_{0}, & C_{1}^{\nabla J}=-\frac{1}{4} W_{0} .
\end{array}
$$

In the HF fits that originally determined the $\mathrm{SkM}^{*}$ parameters, the effects of $C_{t}^{T}$ (the " $J^{2}$ terms") were neglected because of technical difficulties. These terms have often been included in subsequent RPA calculations. To maintain self consistency here, we have set them to zero both in the HFB calculation and in the QRPA.

[1] 2002 NSAC Long-Range Plan, URL http://www.sc.doe.gov/henp/np/nsac/docs/LRP_5547_FINAL.pdf

[2] 2004 NUPECC Long-Range Plan, URL http://www.nupecc.org/lrp02/long_range_plan_2004.pdf

[3] J. Carlson, B. Holstein, X. Ji, G. McLaughlin, B. Müller, W. Nazarewicz, K. Rajagopal, W. Roberts, and X.-N. Wang, A Vision for Nuclear Theory: Report to NSAC; arXiv:nucl-th/0306005

[4] J. Dobaczewski and W. Nazarewicz, Phil. Trans. R. Soc. Lond. A 356, 2007 (1998).

[5] S. Goriely, M. Samyn, P.-H. Heenen, J. Pearson, and F. Tondeur, Phys. Rev. C 66, 024326 (2002).

[6] S. Goriely, M. Samyn, M. Bender, and J. Pearson, Phys. Rev. C 68, 054325 (2003).

[7] M. Stoitsov, J. Dobaczewski, W. Nazarewicz, S. Pittel, and D. Dean, Phys. Rev. C 68, 054312 (2003).

[8] P. Ring and P. Schuck, The Nuclear Many-Body Problem (Springer-Verlag, Berlin, 1980).

[9] S. Shlomo and A. Sanzhur, Phys. Rev. C 65, 044310 (2002).

[10] B. Agrawal, S. Shlomo, and A. Sanzhur, Phys. Rev. C 67, 034314 (2003).

[11] B. Agrawal and S. Shlomo, arXiv:nucl-th/0406013.

[12] D. Rowe, Nuclear Collective Motion, Models and Theory (Mathuen, London, 1970).

[13] M. Waroquier, J. Ryckebusch, J. Moreau, K. Heyde, N. Blasi, and S. van der Werf, Phys. Rep. 148, 249 (1987).

[14] G. Colò, P. Bortignon, D. Sarchi, D. Khoa, E. Khan, and N. V. Giai, Nucl. Phys. A 722, 111c (2003).

[15] J. Engel, M. Bender, J. Dobaczewski, W. Nazarewicz, and R. Surman, Phys. Rev. C 60, 14302 (1999).

[16] M. Bender, J. Dobaczewski, J. Engel, and W. Nazarewicz, Phys. Rev. C 65, 054322 (2002).

[17] G. Giambrone, S. Scheit, F. Barranco, P. Bortignon, G. Colò, D. Sarchi, and E. Vigezzi, Nucl. Phys. A 726, 3 (2003).

[18] S. Shlomo and G. Bertsch, Nucl. Phys. A 243, 507 (1975).

[19] K. Liu and N. V. Giai, Phys. Lett. B 65, 23 (1976).

[20] J. B. Touv, A. Moalem, and S. Shlomo, Nucl. Phys. A 339, 303 (1980).

[21] I. Hamamoto, H. Sagawa, and X. Zhang, Phys. Rev. C 55, 2361 (1997).

[22] I. Hamamoto, H. Sagawa, and X. Zhang, Phys. Rev. C 57, R1064 (1998).

[23] I. Hamamoto and H. Sagawa, Phys. Rev. C 60, 064314 (1999).

[24] I. Hamamoto and H. Sagawa, Phys. Rev. C 62, 024319 (2000).

[25] A. Kolomiets, O. Pochivalov, and S. Shlomo, Phys. Rev. C 61, 034312 (2000).

[26] H. Sagawa, Phys. Rev. C 65, 064314 (2002).

[27] I. Hamamoto and H. Sagawa, Phys. Rev. C 66, 044315 (2002).

[28] S. Shlomo and B. Agrawal, Nucl. Phys. A 722, 98c (2003).

[29] K. Hagino and H. Sagawa, Nucl. Phys. A 695, 82 (2001).

[30] M. Matsuo, Nucl. Phys. A 696, 371 (2001).

[31] M. Matsuo, Prog. Theor. Phys. Suppl. 146, 110 (2002).

[32] E. Khan, N. Sandulescu, M. Grasso, and N. V. Giai, Phys. Rev. C 66, 024309 (2002). 
[33] E. Khan, N. Sandulescu, N. V. Giai, and M. Grasso, Phys. Rev. C 69, 014314 (2004).

[34] S. Goriely and E. Khan, Nucl. Phys. A 706, 217 (2002).

[35] S. Goriely, E. Khan, and M. Samyn, Nucl. Phys. A 739, 331 (2004).

[36] A. Platonov and E. Saperstein, Nucl. Phys. A 486, 63 (1988).

[37] S.Kamerdzhiev, J.Speth, and G.Tertychny, Phys. Rep. 393, 1 (2004).

[38] S. Fayans, A. Platonov, G. Graw, and D. Hofer, Nucl. Phys. A 577, 557 (1994).

[39] D. Horen, G. Satchler, S. Fayans, and E. Trykov, Nucl. Phys. A 600, 193 (1996).

[40] I. Borzov, A. Fayans, and E. Trykov, Nucl. Phys. A 584, 335 (1995).

[41] I. Borzov, A. Fayans, E. Krömer, and D. Zawischa, Z. Phys. A 355, 117 (1996).

[42] S. Kamerdzhiev, R. Liotta, E. Litvinova, and V. Tselyaev, Phys. Rev. C 58, 172 (1998).

[43] S. Kamerdzhiev, E. V. Litvinova, and D. Zawischa, Eur. Phys. J. A 12, 285 (2001).

[44] I. Borzov, Phys. Rev. C 67, 025802 (2003).

[45] S. Kamerdzhiev and E. V. Litvinova, Phys. At. Nucl. 67, 183 (2004).

[46] P. Ring, Z. Ma, N. V. Giai, D. Vretenar, A. Wandelt, and L. Cao, Nucl. Phys. A 694, 249 (2001).

[47] D. Vretenar, N. Paar, P. Ring, and G. Lalazissis, Phys. Rev. C 63, 047301 (2001).

[48] Z. Ma, A. Wandelt, N. V. Giai, D. Vretenar, P. Ring, and L. Cao, Nucl. Phys. A 703, 222 (2002).

[49] D. Vretenar, N. Paar, P. Ring, and T. Niksić, Phys. Rev. C 65, 021301 (2002).

[50] T. Niksić, D. Vretenar, and P. Ring, Phys. Rev. C 66, 064302 (2002).

[51] Z. Ma, L. Cao, N. V. Giai, and P. Ring, Nucl. Phys. A 722, 491c (2003).

[52] D. Vretenar, T. Niksić, N. Paar, and P. Ring, Nucl. Phys. A 731, 281 (2004).

[53] N. Paar, P. Ring, T. Niksić, and D. Vretenar, Phys. Rev. C 67, 034312 (2003).

[54] N. Paar, T. Niksić, D. Vretenar, and P. Ring, Phys. Rev. C 69, 054303 (2004).

[55] T. Nakatsukasa and K. Yabana, Prog. Theor. Phys. Suppl. 146, 447 (2002).

[56] T. Nakatsukasa and K. Yabana, Eur. Phys. J. A 20, 163 (2004).

[57] T. Inakura, M. Yamagami, K. Matsuyanagi, S. Mizutori, H. Imagawa, and Y. Hashimoto, Int. J. Mod. Phys. E E13, 157 (2004).

[58] H. Imagawa and Y. Hashimoto, Phys. Rev. C 67, 037302 (2003).

[59] K. Hagino, N. V. Giai, and H. Sagawa, Nucl. Phys. A 731, 264 (2004).

[60] J. Dobaczewski, W. Nazarewicz, T. Werner, J. Berger, C. Chinn, and J. Dechargé, Phys. Rev. C 53, 2809 (1996).

[61] P. Ring, N. Paar, T. Niksić, and D. Vretenar, Nucl. Phys. A68, 372 (2003).

[62] J. Engel, G. McLaughlin, and C. Volpe, Phys. Rev. D 67, 013005 (2003).

[63] E. Perlińska, S. Rohoziński, J. Dobaczewski, and W. Nazarewicz, Phys. Rev. C 69, 014316 (2004).

[64] J. Dobaczewski, H. Flocard, and J. Treiner, Nucl. Phys. A 422, 103 (1984).

[65] J. Dobaczewski, W. Nazarewicz, and P.-G. Reinhard, Nucl. Phys. A 693, 361 (2001).

[66] K. Bennaceur and J. Dobaczewski, to be submitted to Computer Physics Communications (2004).

[67] J. Terasaki, J. Engel, W. Nazarewicz, and M. Stoitsov, Phys. Rev. C 66, 054313 (2002).

[68] J. Dobaczewski, P. Borycki, W. Nazarewicz, and M. Stoitsov, to be published.

[69] S. Shlomo, V. Kolomietz, and H. Dejbakhsh, Phys. Rev. C 55, 1972 (1997).

[70] J. Bartel, P. Quentin, M. Brack, C. Guet, and H.-B. Håkansson, Nucl. Phys. A 386, 79 (1982).

[71] J. Dobaczewski, W. Nazarewicz, and M. Stoitsov, in The Nuclear Many-Body Problem 2001, eds: W. Nazarewicz and D. Vretenar (Kluwer Academic Pub., Dordrecht, 2002), p. 181.

[72] J. Dobaczewski, M. Stoitsov, and W. Nazarewicz, arXiv:nucl-th/0404077.

[73] N. Tajima, Phys. Rev. C 69, 034305 (2004).

[74] J. Blaizot and G. Ripka, Quantum theory of finite systems (MIT Press, Cambridge Mass., 1986).

[75] M. Harakeh and A. van der Woude, Giant Resonances, Fundamental High-Frequency Modes of Nuclear Excitation (Clarendon Press, Oxford, 2001).

[76] J. Terasaki et al., in preparation (2004).

[77] F. Catara, E. Lanza, M. Nagarajan, and A. Vitturi, Nucl. Phys. A 624, 449 (1997).

[78] H. Sagawa and H. Esbensen, Nucl. Phys. A 693, 448 (2001).

[79] J. Dobaczewski and J. Dudek, Phys. Rev. C 52, 1827 (1995), ibid. C 55, 3177(E) (1997).

[80] M. Bender, P.-H. Heenen, and P.-G. Reinhard, Rev. Mod. Phys. 75, 121 (2003).

[81] J. Slater, Phys. Rev. 81, 385 (1951). 